\title{
Sélection pour la consommation alimentaire moyenne journalière résiduelle chez le porc : impacts sur les caractères et défis pour la filière
}

\author{
H. GILBERT ${ }^{l}$, Y. BILLON ${ }^{2}$, L. BROSSARD ${ }^{3}, J . F A U R E^{3}$, P. GATELLIER ${ }^{4}$, F. GONDRET ${ }^{3}$, E. LABUSSIERE $^{3}$, \\ B. LEBRET ${ }^{3}$, L. LEFAUCHEUR ${ }^{3}$, N. LE FLOCH ${ }^{3}$, I. LOUVEAU ${ }^{3}$, E. MERLOT ${ }^{3}$, M.-C. MEUNIER-SALAÜN ${ }^{3}$, \\ L. MONTAGNE', P. MORMĖDE I, D. RENAUDEAU ${ }^{3}$, J. RIQUET', C. ROGEL-GAILLARD \\ J. VAN MILGEN ${ }^{3}, A$. VINCENT ${ }^{3}, J . N O B L E T^{3}$ \\ ${ }^{\prime}$ GenPhySE, Université de Toulouse, INRA, INPT, INP-ENVT, 31320, Castanet-Tolosan, France \\ 2 GenESI, INRA le Magneraud, 17700, Surgères, France \\ ${ }_{3}$ PEGASE, Agrocampus Ouest, INRA, 35590, Saint-Gilles, France \\ ${ }^{4}$ INRA QuaPA, 63122, Saint-Genès-Champanelle, France \\ ${ }_{5}$ GABI, AgroParisTech, INRA, Université Paris-Saclay, 78350, Jouy-en-Josas, France \\ Courriel : helene.gilbert@inra.fr
}

La sélection pour l'efficacité alimentaire est un enjeu majeur pour la filière porcine. La consommation alimentaire moyenne journalière résiduelle chez le porc en croissance est proposée comme mesure de l'efficacité alimentaire nette. Cette synthèse décrit les impacts de la sélection pour ce critère sur les caractères de production et de reproduction, la physiologie et le métabolisme des animaux, et introduit les futurs défis qui en découlent pour la filière ${ }^{1}$.

L'amélioration de l'efficacité alimentaire est un enjeu majeur pour la filière porcine, sur le plan économique en raison du coût de l'aliment, mais aussi pour réduire l'impact environnemental de la production en diminuant les rejets. Une meilleure efficacité alimentaire permet aussi de limiter la compétition pour l'accès aux ressources entre l'alimentation animale, l'alimentation humaine, l'industrie et la production d'agro-carburants. Un effort important a été réalisé pour améliorer l'efficacité alimentaire au cours des 50 dernières années. Cependant, l'occurrence d'évènements climatiques sévères (sécheresses, inondations) dans les zones majeures de production de céréales a entraîné des fluctuations importantes des prix des matières premières au cours de la dernière décennie, avec une tendance générale à la hausse. Dans ce contexte, le coût alimentaire représente toujours environ $70 \%$ du coût de production du porc actuellement en France (IFIP, Institut de la Filière Porcine).

L'efficacité alimentaire est généralement quantifiée en élevage porcin par son inverse, l'Indice de Consommation (IC), qui est le rapport entre la quantité d'aliment ingérée (Consommation
Moyenne Journalière CMJ) et le gain de poids de l'animal (Gain Moyen Quotidien GMQ) sur une période donnée. L'IC dépend de nombreux facteurs, tels que le stade physiologique et les caractéristiques de l'animal (âge, sexe, génétique), la quantité et la qualité d'aliment disponible ainsi que de l'environnement d'élevage. L'amélioration de l'efficacité alimentaire du porc en croissance a été obtenue pour partie grâce à la prise en compte de ces facteurs dans les plans d'alimentation des animaux : l'indice de consommation est ainsi passé de 3,35 kg d'aliment ingéré par $\mathrm{kg}$ de gain de poids en 1984 à 2,73 en 2014 (données IFIPGTE, 2014). En effet, la composition de la ration (énergie et acides aminés) a été ajustée aux besoins des animaux, et le rationnement quantitatif des porcs en élevages de production s'est généralisé pour réduire l'IC. De plus, l'efficacité alimentaire a été améliorée par la sélection d'animaux maigres et à croissance rapide, qui ont des besoins plus faibles par unité de gain de poids que les animaux plus gras et/ou à croissance lente.

Les relations étroites entre IC et autres performances de production ne permettent pas de séparer les gains génétiques dus à l'amélioration de chacun des facteurs contribuant à une meilleure efficacité alimentaire. En 1963, Koch et al ont proposé le concept d'ingéré résiduel ou Consommation Moyenne Journalière Résiduelle (CMJR), aussi appelé efficacité alimentaire nette, pour évaluer spécifiquement l'efficacité d'utilisation des aliments qui est indépendante des besoins de production de l'animal (cf. encadré 1). La CMJR est calculée comme la différence entre la consommation moyenne journalière observée et celle prédite pour les besoins de production et d'entretien de l'animal pour la période considérée. En pratique, elle est souvent obtenue comme un index combinant les enregistrements de performances pour l'ingestion, la croissance et la composition corporelle des animaux en élevage. Ce critère présente l'avantage de ne pas être, contrairement à l'IC, un rapport entre deux quantités présentes dans les objectifs de sélection. Cet article propose de faire une synthèse des travaux expérimentaux réalisés à l'INRA sur la CMJR chez le porc pendant les 20 dernières années et de leurs implications, et de discuter les nouveaux défis qui en découlent pour la filière porcine. 
Encadré 1. Mesures de l'efficacité alimentaire.

L'efficacité d'utilisation des aliments par les animaux en croissance est classiquement évaluée par le rapport entre le gain de poids et la consommation alimentaire pendant une période donnée. II s'agit d'un indicateur de rendement, compris entre 0 et 1. Dans la pratique, l'inverse de l'efficacité alimentaire, appelé Indice de Consommation (IC), est plus utilisé chez les animaux d'élevage. II traduit l'efficacité de conversion de l'aliment en gain de poids et correspond à une mesure économique du coût alimentaire.

L'IC est fortement corrélé à la vitesse de croissance et à la composition corporelle des animaux, ainsi qu'à leurs besoins d'entretien. En conditions d'élevage classiques, les différences de sexe, de poids, de conditions d'élevage (saison ou épisode sanitaire par exemple) expliquent environ un tiers de la variabilité de l'ingéré chez le porc en croissance. Les facteurs tels que vitesse de croissance et composition corporelle expliquent aussi environ un tiers de cette variabilité. Le dernier tiers n'est pas expliqué par les facteurs identifiables en élevage : il est appelé consommation résiduelle (figure A). A partir de cette décomposition, de nouveaux critères ont été proposés pour travailler sur les composantes de l'efficacité alimentaire, l'IC intégrant toutes les sources de variation sans les distinguer.

Figure A. Relations entre Indice de Consommation (IC), consommations observée et résiduelle.

\begin{tabular}{|c|c|c|}
\hline $\begin{array}{l}\text { gain de poids } \\
\text { composition } \\
\text { du gain }\end{array}$ & $\begin{array}{l}\text { poids } \\
\text { métabolique } \\
\text { moyen }\end{array}$ & $\begin{array}{l}\text { consommation } \\
\text { résiduelle }\end{array}$ \\
\hline $\begin{array}{l}\text { Besoins de } \\
\text { production }\end{array}$ & $\begin{array}{l}\text { Besoins } \\
\text { d'entretien }\end{array}$ & $\begin{array}{l}\text { Autres besoins liés à la digestion, } \\
\text { l'activité , le métabolisme, la santé, } \\
\text { la réponse au stress... }\end{array}$ \\
\hline
\end{tabular}

Le critère le plus étudié est la consommation résiduelle ou Consommation Moyenne Journalière Résiduelle (CMJR) qui représente les 30 à $40 \%$ de la variabilité de l'ingéré du porc en croissance non expliqués. II revient à quantifier une efficacité alimentaire nette des besoins de production des animaux. II s'agit alors de corriger la quantité ingérée réelle par les besoins de production liés à la croissance et par les besoins d'entretien liés au poids métabolique des animaux. Cette correction peut être obtenue par simple régression linéaire multiple de l'ingéré moyen journalier sur la vitesse de croissance (typiquement le gain moyen quotidien), la composition corporelle (typiquement une mesure d'épaisseur de lard aux ultra-sons en fin de croissance, ou une estimation du taux de muscle des pièces à l'abattoir), et le poids métabolique moyen (poids vif moyen de l'animal pendant la période à la puissance 0,60 (Noblet et al 1999)).

Figure B. Relations entre consommation moyenne journalière observée et celle prédite d'après les besoins de production et d'entretien des animaux.

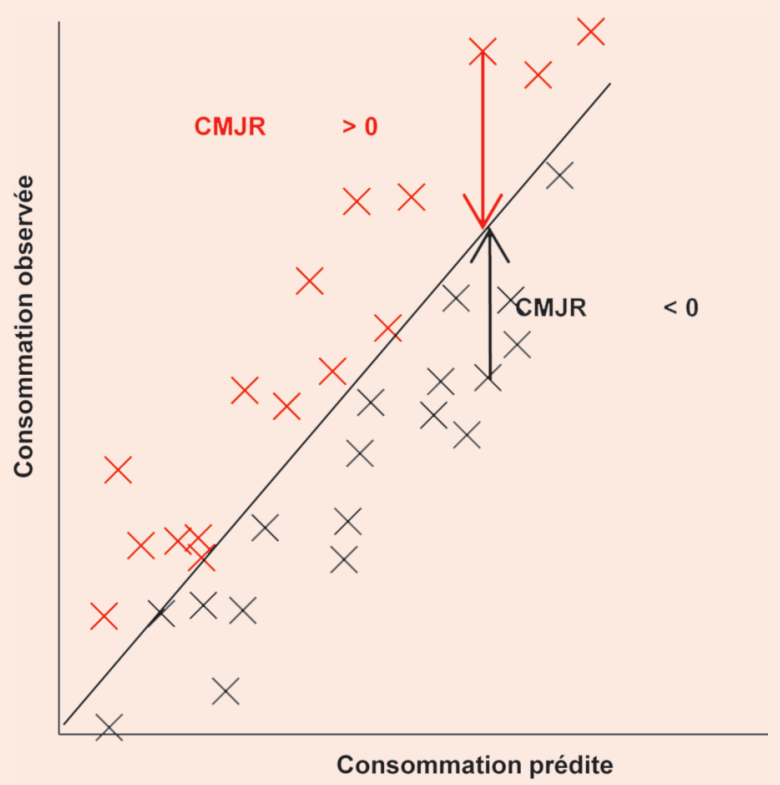

Par construction, la moyenne de la CMJR est nulle. Une CMJR négative correspond à un ingéré plus faible que l'ingéré prédit d'après les performances de l'animal, et donc à un animal plus efficace, alors qu'une CMJR positive correspond à un animal qui ingère plus que la quantité prédite, et donc un animal moins efficace (figure B). 


\section{1 / Génétique de la consom- mation moyenne journalière résiduelle}

Dans l'espèce porcine, des études sont menées depuis 20 ans pour proposer des stratégies d'amélioration de l'efficacité de production en évitant de dégrader les autres paramètres déterminant la durabilité de la production. Ces études s'intéressent à l'efficacité alimentaire dans des populations en sélection (par exemple Saintilan et al 2013) et dans des expérien- ces de sélection divergente sur l'efficacité alimentaire, menées en France par l'INRA en race Large White (Gilbert et al 2007) et aux États-Unis en race Yorkshire (Cai et al 2008). La sélection de lignées divergentes pour un objectif de sélection unique permet d'évaluer les réponses à la sélection sur le caractère cible et les autres caractères intéressant la production (preuve de concept), et d'étudier l'impact de cette sélection sur la physiologie des animaux, servant ainsi de base à la production de connaissances spécifiques en lien avec ce caractère.

\section{1 / Dispositif expérimental}

L'expérience de sélection divergente de l'INRA a été initiée en 1999 à l'unité expérimentale porcine GenESI (Surgères, Charentes) à partir d'une population de 30 mâles de centre d'insémination et 30 femelles Large White. Le détail du processus de sélection et de testage est donné dans l'encadré 2. La sélection a conduit à l'obtention de deux lignées séparées, l'une à CMJR réduite $\mathrm{CMJR}^{-}$, dans laquelle les animaux sont plus efficaces, et l'autre à CMJR élevée $\mathrm{CMJR}^{+}$, dans laquelle les animaux sont moins efficaces.

Encadré 2. Conduite des lignées divergentes « consommation moyenne journalière résiduelle - CMJR ».

À partir d'un troupeau de fondateurs (F0) de 30 truies et de 30 verrats Large White peu apparentés, une première cohorte de 116 candidats à la sélection (G0) a été testée (figure). Les 6 verrats ayant la plus forte CMJR ont été gardés pour initier la lignée $\mathrm{CMJR}^{+}$(excès d'ingéré par rapport aux prédictions) et les 6 verrats ayant la plus faible ont été gardés pour initier la lignée CMJR- (défaut d'ingéré par rapport aux prédictions). Parmi la trentaine de portées nées, 40 couples de pleines sœurs ont été distribués dans deux troupeaux de femelles reproductrices pour initier chaque lignée. La pression de sélection à chaque génération était de $7 \%$. Jusqu'à la génération $\mathrm{G} 5$, à chaque génération chaque truie produisait trois portées : les deux premières avec le même verrat CMJR, la troisième avec un verrat Large White $x$ Piétrain ou Duroc pour des protocoles de détection de QTL. La première portée servait à la sélection des reproducteurs de la génération suivante (testage de la voie mâle uniquement). La deuxième portée servait de contrôle de la réponse à la sélection. Des mesures de croissance, consommation et composition de carcasse et qualité de la viande étaient réalisées. Pour les générations G6 à G9, les premières portées ont servi de support à des programmes expérimentaux dédiés à l'étude d'un ou plusieurs caractères liés à la CMJR, les reproducteurs étant issus des troisièmes (G6, G8 femelles), quatrièmes (G8 mâles et G9) et cinquièmes (G7) mises bas.

Figure. Schéma de la conduite des lignées divergentes pour la CMJR. Les carrés représentent les verrats et les ronds représentent les truies à chaque génération.

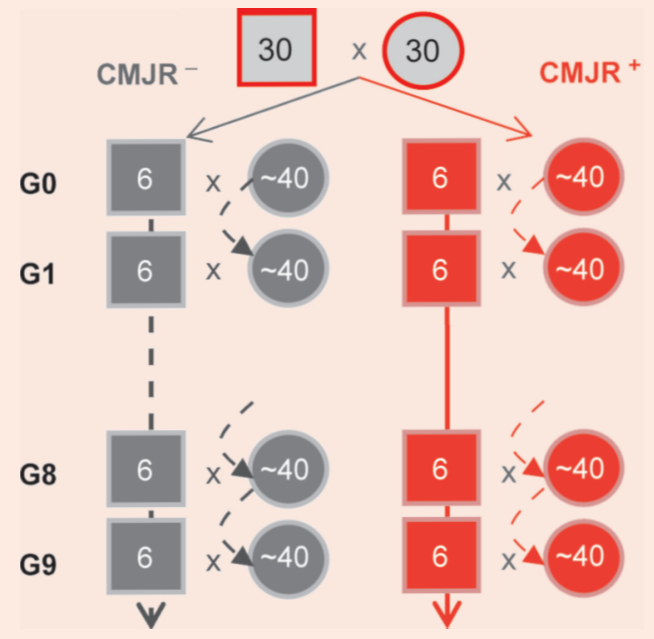

L'index de sélection a été établi pour des porcs mâles entiers Large White entre poids vifs fixes de 35 et $95 \mathrm{~kg}$, alimentés à volonté, élevés en groupe dans des loges équipées d'un automate d'alimentation (Acema 64). L'index est obtenu à partir de la quantité d'aliment consommé (CMJ = consommation moyenne journalière, en g d'aliment/jour), de la vitesse de croissance ( $\mathrm{GMQ}=$ Gain Moyen Quotidien, en g/jour) et d'une estimation de la composition tissulaire du gain de poids à $95 \mathrm{~kg}$ de poids vif par l'épaisseur de lard dorsal obtenue par mesures d'épaisseur de lard dorsal aux ultrasons (ELD, en mm). L'indice de sélection brut a la forme CMJR $=\mathrm{CMJ}-1,06 \mathrm{GMQ}-37 \mathrm{ELD}$. Seuls les mâles sont testés, les femelles sont conduites sans pression de sélection, chaque mère étant remplacée en théorie par une de ses filles à chaque génération pour maintenir la diversité génétique sur la voie femelle.

Le troupeau de truies est distribué pour chaque lignée sur les deux élevages de l'unité expérimentale GenESI, les deux tiers étant localisés à l'unité expérimentale du Magneraud (17700 Surgères) et un tiers à l'unité expérimentale du Rouillé (86480 Rouillé), où se trouvent les automates de contrôle de l'ingéré individuel. Tous les porcelets testés sont donc transférés à Rouillé au sevrage (4 semaines), les porcelets des deux élevages sont regroupés par lignée dans des loges de post-sevrage jusqu'à 10 semaines d'âge. Ils sont alors transférés en loges d'engraissement où ils sont élevés par groupe de 12 par sexe et lignée jusqu'au poids d'abattage ( 110 kg de poids vif, soit 23 à 26 semaines d'âge). A l'abattage, les caractéristiques de carcasse (longueur, poids chaud, poids froid, épaisseur de bardière) sont enregistrées, ainsi que les poids des morceaux de découpe et les caractéristiques de la viande à $24 \mathrm{~h}$ post abattage ( $\mathrm{pH}$, couleur, temps d'imbibition). 
Des phénotypes ont été enregistrés à chaque génération sur les mâles candidats à la sélection, entre 35 et $95 \mathrm{~kg}$ de poids vif, et sur leurs collatéraux femelles et mâles castrés pour les caractères de croissance, entre 10 semaines d'âge et $110 \mathrm{~kg}$ de poids vif, ingestion, composition corporelle in vivo, efficacité alimentaire et, pour les collatéraux uniquement, composition de carcasse et caractéristiques de qualité de la viande enregistrées à l'abattoir. Des performances sont ainsi disponibles pour 3080 animaux de la génération G0 (première génération de sélection) à la génération G9. Les animaux étaient nourris ad libitum avec un mélange de céréales et de tourteau de soja contenant $10 \mathrm{MJ}$ d'énergie nette $/ \mathrm{kg}$ et $160 \mathrm{~g}$ de protéines brutes $/ \mathrm{kg}$, avec un minimum de $0,80 \mathrm{~g}$ de lysine digestible/ MJ d'énergie nette.

\section{2 / Génétique de la CMJR et réponses à la sélection}

a) Réponses à la sélection sur les performances de production et de carcasse

L'héritabilité de la CMJR estimée dans cette expérience de sélection est de $0,13 \pm 0,05$, ce qui est plus faible que d'autres résultats de la littérature $(0,20$ à 0,40, Saintilan et al 2013). La corrélation génétique entre la CMJR et l'IC est estimée à $0,39 \pm 0,12$. Ces deux résultats confirment la possibilité d'améliorer l'efficacité alimentaire des animaux en sélectionnant sur la CMJR. L'estimation des réponses à la sélection sur la CMJR pour les caractères de production majeurs (figure 1) montre une différence de - 165 g/j de CMJR (3,84 écart-types génétiques $\left.\left(\sigma_{\mathrm{g}}\right)\right)$ entre les lignées CMJR- et $\mathrm{CMJR}^{+}$après 10 générations de sélection. De plus, la consommation journalière est réduite $(-270 \mathrm{~g} / \mathrm{j}$ de $\mathrm{CMJ})$, et la différence pour 1'IC atteint - $320 \mathrm{~g}$ d'aliment/ kg de gain de poids. Comme atten$\mathrm{du}$, la vitesse de croissance, mesurée par le GMQ, et la composition corporelle, mesurée par l'Épaisseur de Lard Dorsal in vivo (ELD) ne diffèrent pas significativement entre lignées. Ces résultats montrent que la sélection sur la CMJR permet bien de concentrer la pression de sélection sur la part de l'efficacité alimentaire qui ne dépend pas des performances de production.

Un examen détaillé des réponses à la sélection sur la composition de carcasse montre cependant une augmentation significative sur le taux de viande maigre de la carcasse (figure 1), qui est supérieur de 2,6 points dans la lignée CMJR-. Cette différence s'accompagne d'un meilleur rendement de carcasse et de proportions plus élevées de jambon, longe et épaule, alors que les proportions de panne et de bardière diminuent. Ces réponses

Figure 1. Réponses à la sélection sur la Consommation Moyenne Journalière Résiduelle (CMJR) pour les caractères de croissance, de consommation et de composition de carcasse de la génération G0 à la génération G9, exprimées en écart-types génétiques des caractères.

$\mathrm{CMJR}^{+}=$lignée à CMJR élevée, moins efficace ; CMJR- = lignée à CMJR faible, plus efficace $; \mathrm{CMJ}=$ consommation moyenne journalière $; \mathrm{GMQ}=$ Gain Moyen Quotidien ; IC = Indice de Consommation ; ELD = Épaisseur de Lard Dorsal ; IQV = Indice de Qualité de la Viande ; TVM = Taux de Viande Maigre de la carcasse estimé par combinaison linéaire des poids de morceaux.
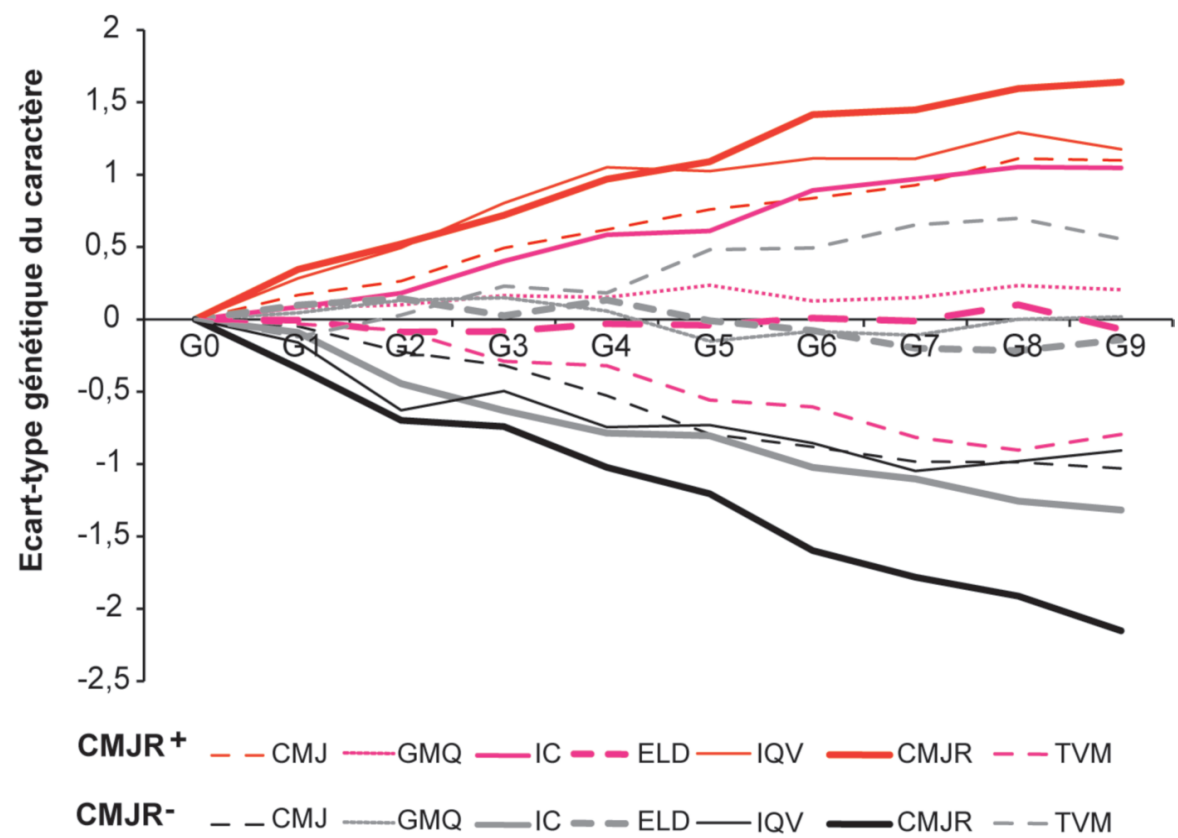

sont dues au fait que la CMJR dans ces lignées est calculée à partir de l'épaisseur de lard dorsal mesurée in vivo en fin de test, qui est une mesure imparfaite et partielle de la composition corporelle (rapport gras/maigre). Le choix du critère de composition corporelle à utiliser pour le calcul de la CMJR est donc crucial pour maîtriser les réponses à la sélection : il doit tenir compte des réponses attendues sur les différents caractères, mais aussi de la facilité et du coût de collecte de l'information sur l'ensemble des animaux à tester. Pour combiner ces contraintes, les mesures d'épaisseur de lard dorsal aux ultrasons, qui peuvent être obtenues in vivo sur de futurs reproducteurs, et le taux de muscle des pièces, estimé en routine dans les abattoirs sur carcasse, sont parmi les meilleures approximations actuellement disponibles sur de grands nombres d'animaux. L'imagerie, de type tomographie ou basée sur des caméras 3D, offrira certainement à termes de nouvelles perspectives d'estimation précise sur animaux entiers vivants.

\section{b) Caractères de qualité de la viande}

Les progrès génétiques sur la vitesse de croissance, la quantité de maigre des carcasses et l'efficacité alimentaire depuis les années 60 ont généralement un impact défavorable sur les caractères de qualité de la viande (Tribout et al 2004). La figure 1 montre que le critère de qualité de la viande IQV, indice de qualité technologique de transformation du jambon cuit combinant $\mathrm{pH}$ ultime, clarté et capacité de rétention d'eau du muscle, a été significativement affecté dès les premières générations de sélection, avec une altération de la qualité technologique de la viande dans la lignée la plus efficace (Gilbert et al 2007, Lefaucheur et al 2011, Faure et al 2013). Cette différence atteint 3,41 points $\left(2,12 \sigma_{\mathrm{g}}\right)$ en génération $\mathrm{G} 9$. Ces réponses sont liées à des modifications du métabolisme énergétique des muscles (voir partie suivante) et des caractéristiques des fibres musculaires : plus de fibres glycolytiques plus grosses ont été observées chez les porcs $\mathrm{CMJR}^{-}$, ce qui conduit à un stockage de glycogène musculaire accru (Lefaucheur et al 2011). On observe également un taux de gras intramusculaire réduit (Lefaucheur et al 2011, Faure et al 2013). Néanmoins, aucun impact significatif sur les paramètres d'oxydation des lipides et des protéines durant la maturation et le stockage de la viande n'a été observé en génération G6 (Gilbert et al 2012a). Des relations génétiques défavorables similaires entre la qualité de la viande et la CMJR ont été rapportées dans les populations porcines commerciales françaises en sélection (Saintilan et al 2013), qui allaient de pair avec des relations défavorables similaires entre qualité de 
la viande et IC. Cette relation n'est pas toujours observée dans d'autres travaux : une première étude sur les lignées divergentes américaines ne montrait pas de différence significative entre lignées (Smith et al 2011), alors qu'Arkfeld et al (2015), après quelques générations supplémentaires, rapportent des pertes en eau accrues et une couleur plus claire de la viande dans la lignée à CMJR élevée.

Finalement, des analyses sensorielles ont montré des différences entre lignées principalement pour l'apparence de la viande (figure 2). Les qualités gustatives étaient faiblement impactées par la sélection, laissant supposer que les différences ne sont probablement pas perceptibles par les consommateurs (Faure et al 2013). Ces résultats montrent que l'utilisation de la CMJR comme critère de sélection pour l'efficacité alimentaire ne permet pas de contourner l'antagonisme génétique entre efficacité alimentaire et qualité technologique de la viande rapporté dans la littérature en s'appuyant sur l'IC. La seule solution pour empêcher la détérioration de la qualité technologique de la viande en réponse à la sélection sur l'efficacité alimentaire demeure donc une contrainte sur ce critère dans les index de sélection, tel que pratiqué actuellement dans les schémas collectifs de sélection porcine en France.

\section{c) Dynamiques de croissance et d'ingestion} et besoins nutritionnels

Des études de l'effet de la sélection sur la dynamique de croissance et d'ingestion des porcs, ainsi que de la cinétique de leurs besoins nutritionnels ont été réalisées. Gilbert et al (2009a) ont montré, à l'aide du logiciel InraPorc ${ }^{\circledR}$ appliqué aux six premières générations de sélection, que la lignée $\mathrm{CMJR}^{-}$a une vitesse de croissance réduite de $7,2 \%$ pendant la croissance par rapport à la lignée $\mathrm{CMJR}^{+}$, associée à une ingestion journalière réduite de $6 \%$ en début de période et de $13 \%$ en fin de contrôle (figure 3A). Ces différences s'accompagnent de besoins en lysine digestible relativement à l'énergie nette plus élevés chez les porcs $\mathrm{CMJR}^{-}$, de $+7 \%$ à 10 semaines d'âge à + $20 \%$ à l'âge d'abattage (figure 3B), différences confirmées expérimentalement (Brossard et al 2012a). Les paramètres descripteurs des cinétiques de croissance et de consommation pour chaque porc ont été analysés (Gilbert et al 2009a). Le paramètre représentant la $\mathrm{CMJ}$ à $50 \mathrm{~kg}$ de poids vif a été identifié comme un prédicteur précoce possible de la CMJR : il est héritable $\left(\mathrm{h}^{2}=0,41\right)$ et a une corrélation génétique de 0,61 avec la CMJR. Des différences de cinétiques ont aussi été rapportées dans les lignées divergentes américaines, en particulier pendant la phase tardive de croissance (Cai et al

Figure 2. Qualité sensorielle de la viande (longe) évaluée sur une échelle de 0 à 10 (absent - très fort) chez des porcs CMJR- $(n=60)$ et des porcs $\mathrm{CMJR}^{+}(n=57)$ après 7 générations de sélection ( $\left.t: P<0,1 ;{ }^{* *}: P<0,01 ;{ }^{* * *}: P<0,001\right)$, (d'après Faure et al 2013).
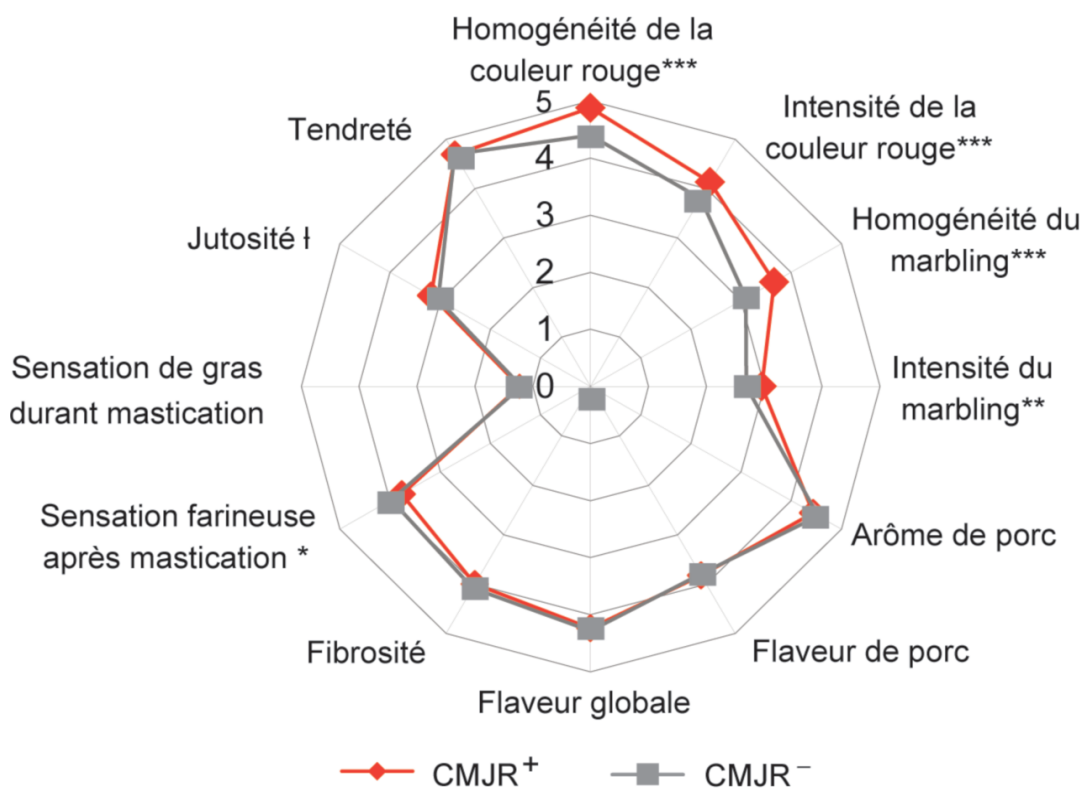

Figure 3. Consommation moyenne journalière $A$ ) et besoins en lysine digestible $B$ ) en fonction de l'âge du porc en croissance dans la lignée CMJR- (en gris) et la lignée $\mathrm{CMJR}^{+}$(en rouge) à la deuxième (G1, tirets) et sixième (G5, traits pleins) génération de sélection (adapté de Gilbert et al 2009).

A

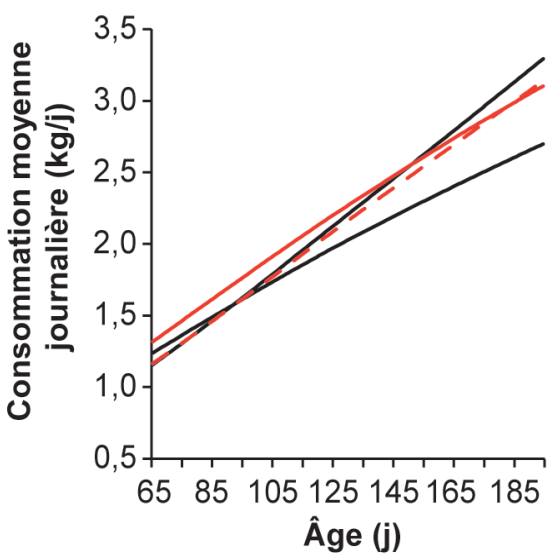

2011, 2012), et dans les lignées commerciales françaises (Saintilan et al 2015). Ainsi, la sélection pour la CMJR modifie les dynamiques d'ingestion des porcs, ainsi que leurs besoins nutritionnels au cours de la période d'engraissement. Les estimations obtenues suggèrent que les animaux les plus efficaces peuvent être carencés en acides aminés en début de croissance avec les aliments commerciaux tels que formulés actuellement. Ces estimations ont été réalisées sur des femelles et mâles castrés, mais il est vraisemblable que le phénomène sera plus marqué sur des animaux mâles entiers, type sexuel naturellement plus maigre et plus efficace que les femelles et les mâles castrés. Les besoins nutritionnels doivent donc être réévalués pour ajuster au mieux les rations délivrées aux ani-
B

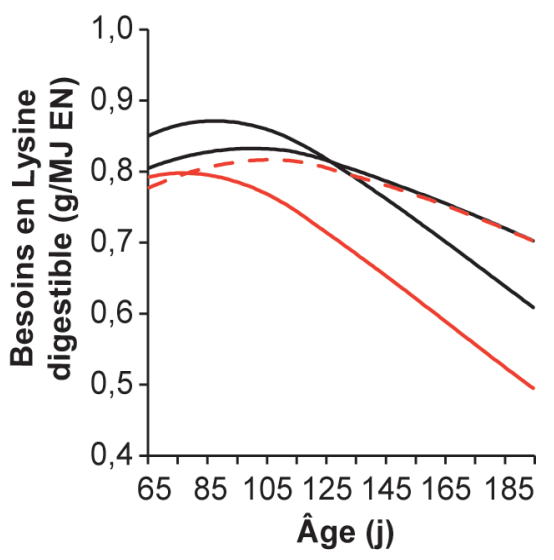

maux sélectionnés pour une meilleure efficacité alimentaire. Couvrir ces besoins peut passer par l'incorporation accrue de matières premières coûteuses, ou par la supplémentation des rations en acides aminés de synthèse. L'impact économique de ces stratégies devra être évalué de façon globale à l'échelle des élevages, en particulier en fonction des ressources alimentaires disponibles et de leurs coûts, et des performances de production associées.

\section{d) Excrétion d'azote et de phosphore}

Une diminution de l'impact environnemental est attendue en réponse à l'amélioration de l'efficacité alimentaire des animaux. Une diminution de l'excrétion de l'azote et du phosphore chez les porcs 
$\mathrm{CMJR}^{-}$après six générations de sélection (4 et $2,4 \%$, respectivement) a été estimée par modélisation (figure 4, Faure et al 2012) et confirmée sur des animaux de la génération suivante soumis à un challenge de chaleur (Renaudeau et al 2013). Ces différences n'étaient pas nécessairement retrouvées chez des animaux des générations précédentes logés individuellement (Barea et al 2010) ou de même génération plus jeunes soumis à un challenge inflammatoire (Labussière et al 2015). Des études sur des populations commerciales françaises (Saintilan et al 2013) et expérimentales (Shirali et al 2012, 2014) ont confirmé des corrélations génétiques fortes et favorables entre l'excrétion et l'efficacité alimentaire, allant de 0,38 avec la CMJR à 0,99 avec l'IC. Ces études portent sur l'évaluation du potentiel d'excrétion des animaux nourris avec un régime alimentaire unique couvrant largement leurs besoins. Pour aller plus loin, il faudrait évaluer les réponses à des régimes alimentaires ajustés à leurs besoins nutritionnels : cela pourrait révéler des différences additionnelles de capacité de rétention. Il est aussi possible que des interactions entre la composition de la ration en acides aminés et le stress en élevage modifient ces résultats, certains stress, tels que l'inflammation, entraînant un besoin accru en acides aminés.

\section{3 / Marqueurs génétiques de la CMJR}

Si l'ingéré peut maintenant être mesuré individuellement, y compris pour des porcs en groupe, cela reste un processus coûteux lié à l'utilisation d'automates d'alimentation. Les outils moléculaires permettent l'identification de régions du génome impliquées dans le déterminisme des caractères (QTL pour quantitative trait loci) dont les polymorphismes peuvent être utilisés comme prédicteurs des caractères dans les schémas de sélection. Cependant, si les analyses génomiques des lignées divergentes ont montré une évolution du génome en lien avec la sélection (figure 5A), très peu de QTL ont été identifiés pour la CMJR, et avec des niveaux de signification faibles, alors que des niveaux de signification plus élevés étaient atteints pour les autres caractères de production (figure 5B) (Riquet et al 2014). Certaines de ces régions sont également détectées dans d'autres études chez le porc (Onteru et al 2013, Fontanesi et al 2014) ou dans des régions homologues bovines (Santana et al 2014, Rolf et al 2012). Cependant, l'absence de région chromosomique avec un effet important sur la CMJR est un résultat concordant avec la littérature. Elle suggère l'implication de plusieurs voies biologiques dans la variation de l'efficacité alimentaire et une diversité

Figure 4. Utilisation des éléments $N, P$ et $K$ : simulation de la part excrétée (urine, fèces) dans la lignée CMJR- $(n=50)$ et la lignée $\operatorname{CMJR}^{+}(n=48)$ après 7 générations de sélection ( $P<0,001$ pour les 3 éléments) (exprimée en \% des éléments consommés), (d'après Faure et al 2012).

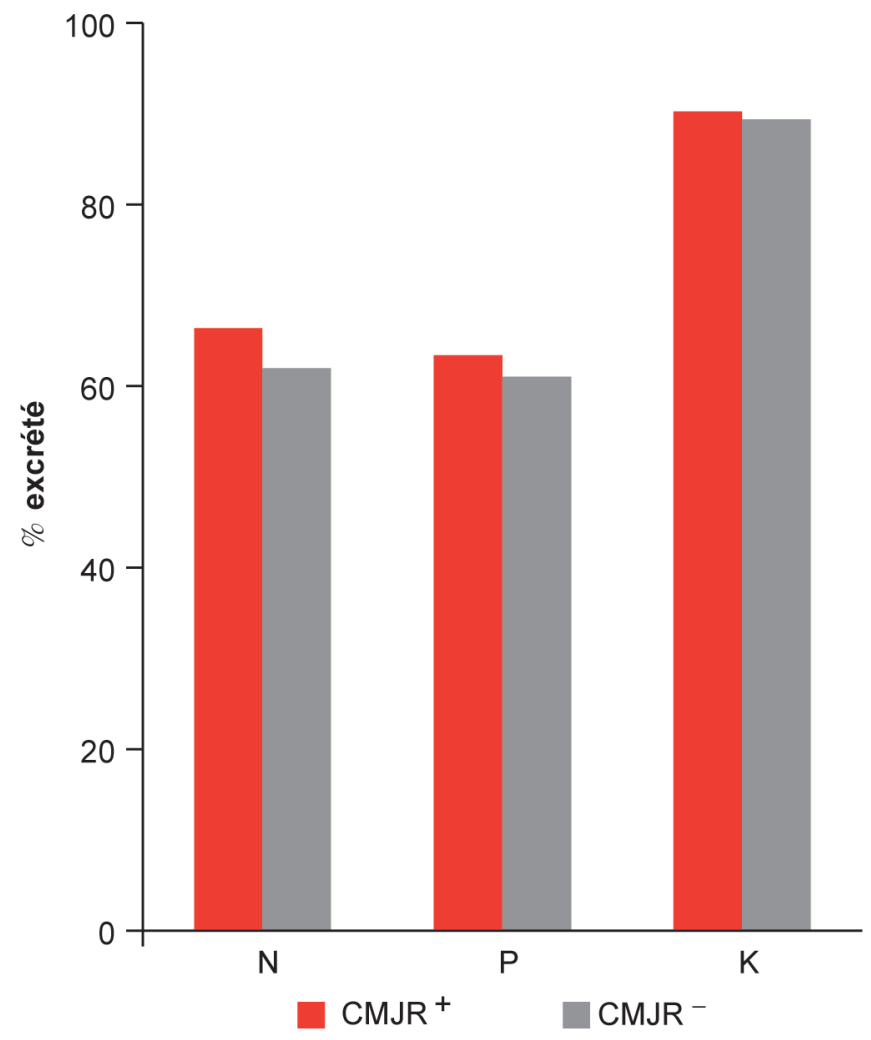


Figure 5. A) Évolution de la structure génomique de la lignée CMJR- $(n=149)$ et de la lignée $\mathrm{CMJR}^{+}(n=121)$ entre la génération $\mathrm{G} 0$ et la génération G6.

B) Distribution des régions chromosomiques détectées à un niveau suggestif (en italique) ou significatif (en gras) pour chaque groupe de caractères : croissance, ingestion et efficacité alimentaire en rouge, composition de carcasse en noir, qualité de la viande en gris ; GMQ = Gain Moyen Quotidien ; CMJ = Consommation Moyenne Journalière $; I C=$ Indice de Consommation ; CMJR = Consommation Moyenne Journalière Résiduelle ; ELD = Épaisseur de Lard Dorsal ; Longueur = Longueur de la carcasse $;$ TVM = Taux de Viande Maigre de la carcasse $; R D T=$ Rendement de carcasse froide $;$ Hachage $=$ poids de d'épaule $;$ Poitrine $=$ poids de poitrine $;$ Longe = poids de longe ; $B A R D=$ poids de bardière; $a, b, L=$ coordonnées $L^{*}, a^{*}, b^{*}$ mesurant la couleur de la viande mesurée à l'abattoir sur les muscles Gluteus medius et Gluteus supeficialis ; $p H u=p H$ de la viande 24 h après abattage; IMB = temps d'imbibition de $1 \mathrm{~cm}^{2}$ de papier $\mathrm{pH}$ apposé sur le muscle Gluteus medius (adapté de Riquet et al 2014).

A)

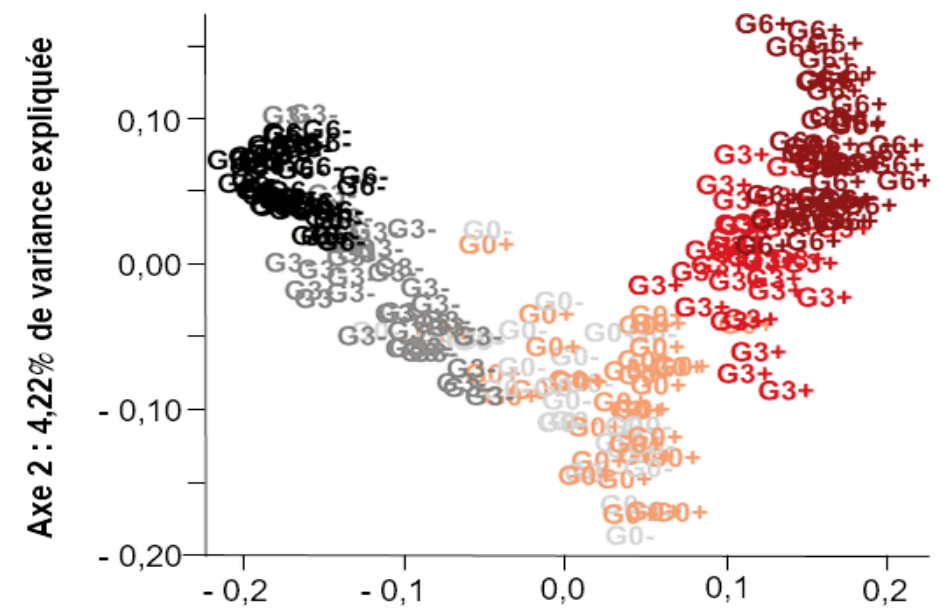

Axe $1: 12,37 \%$ de variance expliquée

B)

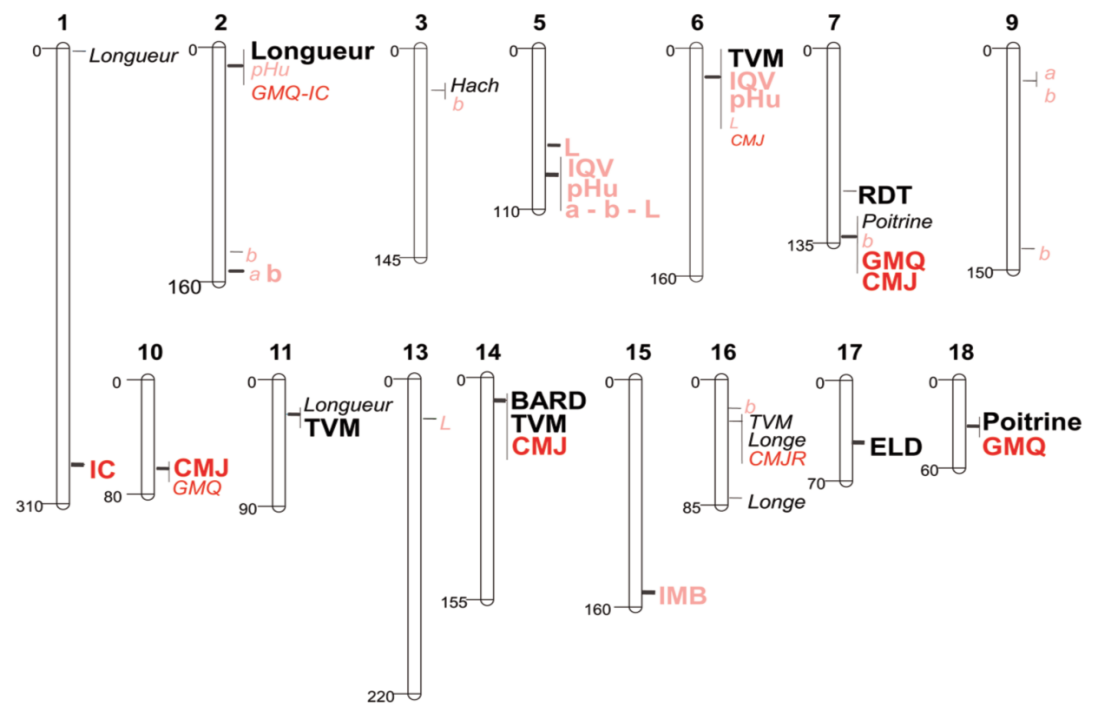

Figure 6. Schéma synthétique du devenir des aliments dans l'organisme.

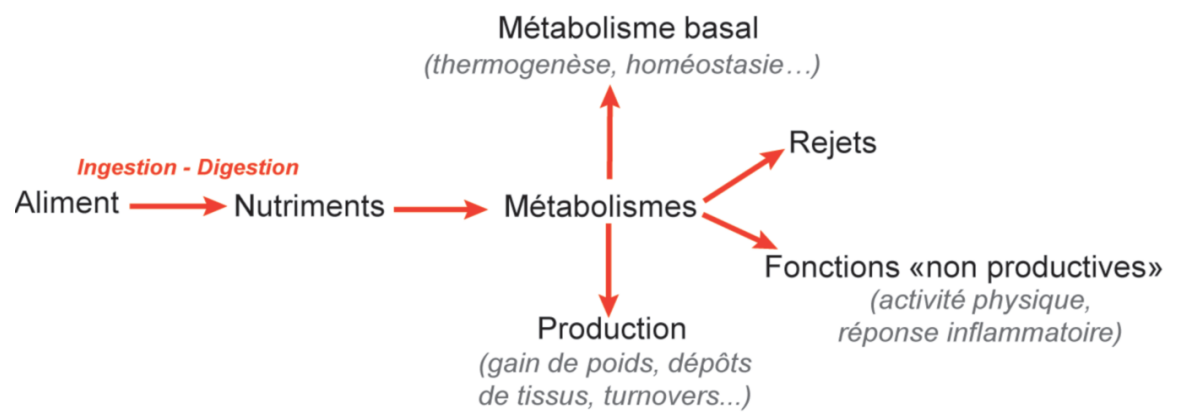

ne sont retrouvées qu'avec un régime riche en fibres et en énergie dans les générations plus avancées (Mauch et al 2015).

\section{2 / Métabolismes énergétique et protéique}

L'étude du métabolisme énergétique a été menée sur l'animal entier par des mesures en chambre respiratoire, et à l'échelle des tissus importants dans l'homéostasie énergétique (foie, muscle, tissus adipeux) en combinant des approches ciblées (activités d'enzymes clés) et sans a priori (protéomique, transcriptomique). Cela a permis de mettre en évidence les processus pouvant jouer un rôle majeur dans l'efficacité des porcs $\mathrm{CMJR}^{-}$dans différents contextes. Il en ressort que les porcs $\mathrm{CMJR}^{-}$privilégieraient une stratégie de production d'énergie « à court-terme " dans le muscle, centrée sur le stockage du glucose en glycogène, puis son utilisation (Le Naou et al 2012, Faure et al 2013, Labussière et al 2015, Vincent et al 2015, Gondret et al 2017). À l'inverse, les porcs $\mathrm{CMJR}^{+}$ privilégieraient une stratégie basée sur le stockage du glucose sous forme de lipides, et leur utilisation via l'oxydation des acides gras dans les mitochondries. Ces modifications sont en lien avec une augmentation de l'activité de l'AMPK (protéine kinase activée par l'AMP) dans le muscle des porcs $\mathrm{CMJR}^{+}$, senseur du déficit énergétique cellulaire qui stimule les voies cataboliques productrices d'énergie (oxydation des acides gras, glycogénolyse, glycolyse) (Faure et al 2013). Une réduction du stress oxydant dans le muscle et le foie des porcs CMJRa été également suggérée, sur la base d'une moindre activation de systèmes enzymatiques antioxydants (Vincent et al 2015, Gondret et al 2017) et d'une production de ROS (espèces réactives à l'oxygène) réduite dans les mitochondries isolées de muscle des lignées américaines (Grubbs et al 2013). Cependant, aucune différence d'expression des protéines découplantes de l'énergie n'a été observée dans le muscle (Lefaucheur et al 2011). Des différences d'activités enzymatiques et d'expression de plusieurs gènes suggèrent une augmentation des capacités lipolytiques et de l'oxydation mitochondriale des acides gras dans le tissu adipeux sous-cutané des porcs CMJR- (Gondret et al 2014, Louveau et al 2016, Gondret et al 2017). Ces modifications pourraient expliquer la moindre adiposité des porcs $\mathrm{CMJR}^{-}$, mais aussi une meilleure capacité à mobiliser leurs réserves lors de challenges physiologiques (voir § 3).

Les différences de métabolisme protéique des tissus associées à la divergence de CMJR sont moins claires, les résultats variant selon les âges et stades métaboliques considérés et les métho- 
dologies employées. Ainsi, aucune différence d'utilisation de l'azote (\% d'azote absorbé) n'a été rapportée entre lignées à la sixième génération de sélection (Barea et al 2010), alors qu'à la génération suivante, des taux d'utilisation et des dépôts protéiques plus élevés ont été observés pour les porcs CMJR- durant la phase de croissance (Renaudeau et al 2013) mais pas durant la période de post-sevrage (Labussière et al 2015). Par ailleurs, des taux de synthèse protéique et des niveaux d'expression de marqueurs de la synthèse protéique similaires dans les deux lignées divergentes ont été rapportés dans les expériences de sélection menées en France (Le Naou et al 2012) et aux États-Unis (Cruzen et al 2013), mais Vincent et al (2015) et Gondret et al (2017) ont mis en évidence une surexpression de gènes codant pour des facteurs de traduction ribosomale dans le tissu musculaire (longe) des porcs $\mathrm{CMJR}^{-}$en fin de croissance. Les travaux sur les lignées américaines suggèrent un catabolisme protéique réduit chez les animaux CMJRaux alentours de $68 \mathrm{~kg}$ de poids vif, mais aucune différence dans ce sens n'a été trouvée en post-sevrage ou au poids commercial d'abattage $(\sim 115 \mathrm{~kg})$ sur les lignées françaises (Le Naou et al 2012). Finalement, l'expression des gènes impliqués dans la protéolyse dans le muscle serait soit similaire (Vincent et al 2015) soit supérieure (Gondret et al 2017) chez les porcs CMJR-. Des investigations complémentaires sont nécessaires pour préciser la contribution des modifications du métabolisme protéique aux différences d'efficacité alimentaire.

\section{3 / Métabolisme basal}

Une part importante de la différence de besoins énergétiques entre lignées, à alimentation et environnement d'élevage donnés, est due à des différences de métabolisme basal. Chez le porc en croissance, celui-ci est quantifié à travers l'estimation de la production de chaleur à jeun (fasting heat production). Chez des porcs de $60 \mathrm{~kg}$ de poids vif, une réduction moyenne de $10 \%$ de la production de chaleur à jeun à la $6^{\text {ème }}$ génération de sélection chez les porcs CMJR- a été mise en évidence (Barea et al 2010), différence cohérente avec les résultats obtenus sur les lignées américaines (Boddicker et al 2011). Cet effet avait auparavant été rapporté chez la poule pondeuse (Gabarrou et al 1997, Swennen et al 2007). Cela pourrait s'expliquer par les différences métaboliques observées dans les tissus, ou par les différences de stress oxydant. Les différences de poids des viscères observées entre lignées (voir Digestion et aliments fibreux) pourraient également contribuer à diminuer les besoins d'entretien des porcs CMJRCependant, la différence d'ingéré spontané entre lignées ne contribue qu'à
$25 \%$ de la différence des besoins d'entretien observée (Labussière et al 2015), et d'autres facteurs restent donc à identifier.

\section{4 / Activité et comportement alimentaire}

L'activité physique est une des fonctions non directement productives qui contribue fortement à l'utilisation de l'énergie chez le porc. Les porcs CMJRpassent moins de temps debout (- $35 \mathrm{~min}$ debout par rapport aux $\mathrm{CMJR}^{+}$, i.e. $-21 \%$ en génération G6), ce qui contribue à une réduction de l'activité physique générale avec une tendance à la diminution des interactions sociales et des activités d'investigation dans la loge (MeunierSalaün et al 2014). Cette activité réduite représente $14 \%$ de la différence d'ingéré d'énergie métabolisable entre lignées à cette génération (Meunier-Salaün et al 2014), ce qui est cohérent avec les différences de production de chaleur liée à l'activité estimées précédemment (Barea et al 2010). Une différence similaire a été décrite dans les lignées porcines américaines (Sadler et al 2011), et auparavant dans d'autres espèces (volailles Luiting et al 1994 ; souris Bunger et al 1998 ; bovins allaitants Herd et Arthur 2009). De façon corrélée, l'activité alimentaire des porcs CMJR- est réduite, avec un temps d'ingestion journalier plus faible, moins de visites à la mangeoire et une vitesse d'ingestion accrue (Gilbert et al 2009b, Meunier-Salaün et al 2014), des tendances similaires étant observées dans les lignées américaines (Young et al 2011). L'amplitude de ces différences peut être affectée par la composition des groupes (regroupement par lignée et sexe dans les lignées INRA), mais l'activité physique reste un contributeur majeur de l'efficacité alimentaire du porc en croissance. On observe enfin plus de défauts d'aplombs chez les porcs de la lignée $\mathrm{CMJR}^{+}$, potentiellement liés à leur activité physique accrue (MeunierSalaün et al 2014).

\section{5 / Marqueurs biologiques de la CMJR}

L'identification de biomarqueurs mesurables tôt dans la vie de l'animal est recherchée pour prédire l'efficacité alimentaire individuelle, caractère dont la valeur n'est connue qu'à la fin de la période de croissance. Le sang est un compartiment intéressant car il s'agit d'un fluide abondant chez l'animal et son prélèvement est possible à n'importe quelle période de sa vie. Des molécules connues pour être impliquées dans la régulation de la croissance et de l'ingéré alimentaire ont dans un premier temps été testées. La concentration circulante d'IGF-I, un facteur de croissance jouant un rôle essentiel dans le développement et la croissance cellulaires (Le Roith et al 2001), a par exemple été dosée à différents stades de croissance des animaux. Des corrélations génétiques fortes ont été mises en évidence entre la concentration plasmatique d'IGF-I juvénile (environ 10 jours après sevrage) et l'efficacité alimentaire dans des populations commerciales (Bunter et al 2005), puis dans les lignées divergentes américaines (Bunter et al 2010), et des différences phénotypiques ont été trouvées entre les lignées INRA (Gilbert et al 2017b). Ces différences ne sont pas retrouvées chez les animaux au poids commercial d'abattage (Lefaucheur et al 2011, Le Naou et al 2012). La leptine, hormone sécrétée par le tissu adipeux et impliquée dans la régulation de la satiété, a aussi été considérée mais les résultats varient, sans doute en lien avec les différences associées à l'adiposité corporelle entre les expériences (Lefaucheur et al 2011, Hoque et al 2009, Jégou et al 2016a).

L'alternative à ces approches ciblées est l'identification de molécules discriminant les niveaux d'efficacité alimentaire grâce à des méthodes haut-débit sans a priori. L'étude des profils métabolomiques plasmatiques n'a pas permis de dégager de marqueurs majeurs sur des porcs de 132 jours d'âge (Jégou et al 2016b). En revanche, les études réalisées à l'INRA et aux États-Unis montrent que les profils transcriptomiques sanguins des animaux de 35 ou 132 jours d'âge diffèrent de façon majeure entre les deux lignées (Jégou et al 2016b, Liu et al 2015). Les différences les plus marquées sont trouvées pour l'expression de gènes impliqués dans le système immunitaire et la traduction. Un dernier type d'approche basé sur la comparaison des profils protéomiques entre lignées (Grubbs et al 2014, 2016) montre que les protéines sériques peuvent aussi constituer des marqueurs potentiels.

Avant d'être utilisés comme biomarqueurs en production, de tels résultats doivent être validés sur un nombre important d'animaux d'élevage, et les relations avec les autres caractères intéressant la production doivent être estimées pour éviter d'entraîner des effets défavorables non identifiés sur les caractères d'intérêt, ou dans des situations d'élevage alternatives (stress, statut physiologique différent).

\section{3 / Stress, robustesse et consommation moyenne journalière résiduelle}

La sélection pour la CMJR a donc des impacts sur les fonctions de production comme la croissance et les fonctions nonproductives d'animaux élevés en conditions neutres. Ces différences peuvent 
diminuer les capacités des animaux à utiliser les nutriments pour des fonctions de défense ou de réponse au stress, et donc affecter leur robustesse (Knap 2009, Hermesch et al 2015). Une évaluation globale de la survie de 10 semaines d'âge jusqu'à l'abattage des porcs des deux lignées a cependant montré un taux d'élimination pour cause de morbidité et mortalité réduit de 1,8 fois dans la lignée $\mathrm{CMJR}^{-}$par rapport à la $\mathrm{CMJR}^{+}$(Pastorelli et al 2015).

\section{1 / Réponse à un challenge inflammatoire}

Les réponses inflammatoires induisent des modifications métaboliques conduisant les animaux à utiliser leurs nutriments pour les fonctions de défense au détriment des fonctions de production. En réponse à un challenge inflammatoire, les porcelets des deux lignées ont montré des réponses globalement similaires en termes de symptômes (appétit réduit, hyperthermie) et de marqueurs biologiques de l'inflammation (augmentation de l'haptoglobine sanguine et de l'interféron y) (Merlot et al 2011). En revanche, l'expression des marqueurs de l'inflammation dans les tissus impliqués dans la réponse inflammatoire (poumons et ganglions lymphatiques) était plus faible chez les animaux CMJR- une semaine après le challenge. Les deux lignées avaient aussi des performances de croissance et une utilisation de l'énergie de l'aliment similaires dans les jours suivant le challenge (Labussière et al 2015 ; Merlot et al 2016). L'étude des réponses des métabolismes énergétiques et protéiques montre que les porcs CMJRréorientent les nutriments des voies anaboliques vers des voies cataboliques dans les deux jours suivant le challenge (Labussière et al 2015). Cependant, huit jours après le début du challenge, durant la phase chronique de la réponse inflammatoire où le catabolisme musculaire est accru, le dépôt protéique semble mieux se maintenir chez ces mêmes porcs, suggérant une meilleure résilience (Merlot et al 2016). La sélection divergente semble donc avoir entraîné des différences dans les voies métaboliques recrutées par les animaux pour répondre à l'inflammation, mais qui ne conduisent pas, au moins chez le jeune porc, à un désavantage adaptatif pour les animaux les plus efficaces. Cette meilleure tolérance des porcs $\mathrm{CMJR}^{-}$à un challenge immunitaire semble se confirmer en engraissement (Chatelet et al 2017).

\section{2 / Réponse à un challenge thermique}

En conditions de stress thermique, la capacité des animaux à maintenir leur homéostasie est dépendante de leur capacité à perdre de la chaleur et/ou à
Figure 7. Effets de la lignée CMJR et de l'augmentation de la température ambiante sur la température rectale et le rythme respiratoire des porcs.

Les réponses sont modélisées pour chaque caractère par lignée selon les paramètres publiés par Campos et al (2014). Seul le paramètre th1, indicateur de la durée (en jours) de la première phase des réponses d'acclimatation, pour la température rectale est significativement différent entre lignées $(0,85$ jour en CMJR- versus 1,88 jour en $\left.\mathrm{CMJR}^{+}, \mathrm{P}<0,05\right)$.
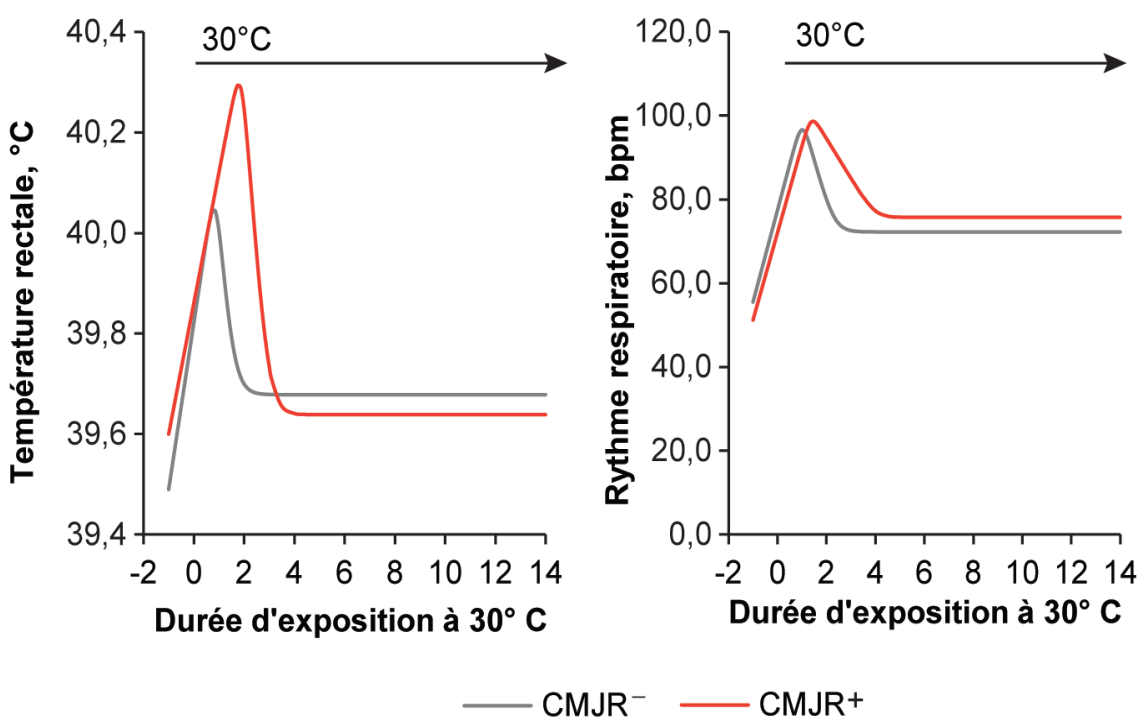

réduire leur production de chaleur métabolique. La moindre production de chaleur liée au métabolisme basal chez les animaux $\mathrm{CMJR}^{-}$constitue potentiellement un élément favorable. Deux challenges thermiques expérimentaux ont permis d'évaluer les réponses de thermorégulation des lignées divergentes. La baisse d'ingéré chez les porcs CMJRsuite à l'élévation de la température à 30 ou $32^{\circ} \mathrm{C}$ était plus faible que chez les $\mathrm{CMJR}^{+}$. Par ailleurs, l'initiation des réponses d'adaptation était plus rapide dans la lignée $\mathrm{CMJR}^{-}$, comme l'indiquent les évolutions des températures rectales et du rythme respiratoire dans les deux lignées (figure 7). Ces dynamiques favorables n'étaient pas accompagnées de différences de concentrations hormonales dans le sang ou de métabolisme énergétique durant l'adaptation (Campos et al 2014), ni de capacité à dissiper la chaleur (Renaudeau et al 2013). En revanche, les porcs $\mathrm{CMJR}^{+}$avaient une capacité accrue à perdre de la chaleur par la voie évaporative, potentiellement en lien avec une production de chaleur et une ingestion d'eau supérieures, ce qui finalement ne permettait pas de dégager un avantage particulier pour l'une ou l'autre lignée. La comparaison des performances des lignées élevées en conditions tropicales humides en $\mathrm{G} 7$ a mis en évidence une vitesse de croissance réduite pendant le test dans la lignée $\mathrm{CMJR}^{-}$, suite à une croissance plus fortement réduite par la chaleur après le sevrage (Gilbert et al 2012b), suggérant à nouveau des stratégies de réponse au challenge thermique différentes entre les lignées. Dans ces conditions d'élevage, l'avantage des animaux $\mathrm{CMJR}^{-}$par rapport aux $\mathrm{CMJR}^{+}$en termes d'efficacité alimentaire était cependant maintenu.

\section{3 / Réponse à un changement de statut physiologique : exemple de la lactation}

La diminution de l'ingéré volontaire des porcs en croissance en réponse à la sélection peut avoir un impact défavorable sur les performances de reproduction et la longévité de la truie en lactation, qui doit utiliser ses ressources corporelles pour répondre aux besoins nutritionnels de sa portée (Prunier et al 2010). En dépit de corrélations génétiques faibles entre CMJR en croissance et caractères de reproduction (Gilbert et al 2012 c), des réponses significatives à la sélection ont été mises en évidence après sept générations de sélection (figure 8). Dans la lignée $\mathrm{CMJR}^{-}$, les truies en lactation avaient un ingéré réduit, des pertes de poids et d'épaisseur de lard dorsal et des poids de portée à $21 \mathrm{j}$ de lactation plus élevés, et sevraient 0,6 porcelets de plus par portée, sans différence significative de poids individuel des porcelets au sevrage (28 j). Young et al (2016) ont rapporté des différences similaires entre les truies des lignées américaines. Aucun problème de fertilité associé à ces réponses en lactation n'a pu être mis en évidence, étant donné le faible nombre de ration $\mathrm{G} 7$ ont par ailleurs été élevées en conditions tropicales (Renaudeau et al 2014), où des différences accrues entre lignées ont été mises en évidence, en problèmes de retour en chaleur observé dans cette étude. Des truies de la géné- 
Figure 8. Réponses corrélées sur les caractères mesurés en lactation sur la truie (ingestion, perte de poids et d'épaisseur de lard, CMJR en lactation) et la portée (croissance naissance - 21 j) suite à la sélection divergente pour la CMJR chez le porc en croissance après 7 générations de sélection (adapté de Gilbert et al 2012c).

$\mathrm{CMJR}^{-}=\mathrm{CMJR}$ réduite $; \mathrm{CMJR}^{+}=\mathrm{CMJR}$ élevée. Toutes les différences entre lignées sont significatives à $\mathrm{P}<0,001$.
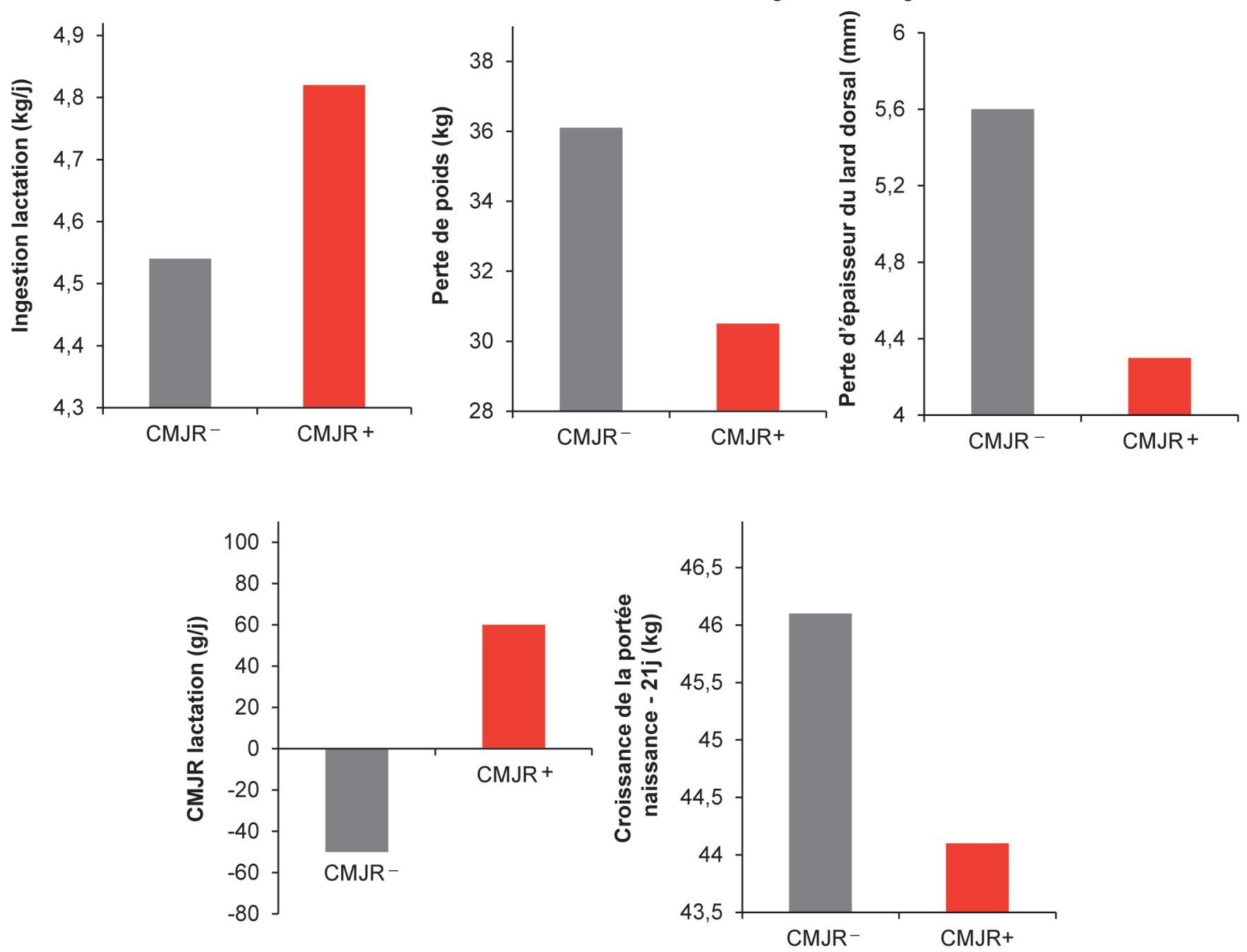

raison de la réduction d'ingéré spontané des truies en réponse à la chaleur et l'humidité. Ces deux études montrent que la sélection pour une CMJR réduite pendant la croissance n'a pas d'impact défavorable sur les performances de la truie en lactation. L'application du concept de CMJR à la lactation a finalement permis de montrer que les porcs $\mathrm{CMJR}^{-}$sont aussi plus efficaces durant la lactation (Gilbert et al 2012c). Cela correspond à une meilleure capacité des truies CMJRà produire du lait et assurer la survie de leur portée en dépit d'une ingestion plus faible en lactation et suggère une robustesse accrue.

L'ensemble de ces résultats amène à s'interroger sur les stratégies habituelles de sélection et de conduite des truies : la sélection pour une augmentation simple de l'ingéré volontaire ne produit pas nécessairement des truies dirigeant plus efficacement leurs ressources vers leur portée, et les truies globalement les plus efficaces semblent être celles qui mobi- lisent et reconstruisent le mieux leurs réserves. Pour aller plus loin sur ce volet, l'examen de la carrière des truies depuis leur mise à la reproduction jusqu'à leur réforme permettrait de proposer des critères de sélection qui intègrent une notion d'efficacité globale et n'entraînent pas la détérioration de l'aptitude des femelles à la reproduction. Un suivi des lactations successives permettra de mieux comprendre les mécanismes biologiques sous-jacents et de proposer des critères de robustesse et de longévité des truies.

\section{4 / Défis pour l'amélioration de l'efficacité alimentaire}

Ces résultats montrent que la sélection pour une meilleure efficacité alimentaire en utilisant la CMJR comme caractère de sélection induit une réduction de l'ingéré volontaire des porcs et de leur consommation d'eau, diminuant les quantités d'intrants utilisées en élevage (figure 9). Elle conduit à la production de porcs plus maigres et présentant un métabolisme énergétique plus efficace, en induisant une réduction significative du métabolisme basal et du niveau d'activité physique des animaux sans modifier leur efficacité digestive. La dégradation des paramètres de qualité technologique de la viande ne semble pas accroître la production de viandes présentant d'importants défauts, mais reste un point de vigilance dans les stratégies de sélection pour l'efficacité alimentaire, de même que la composition nutritionnelle de la ration alimentaire. De plus, la capacité de réponse à des stress ou des changements de conditions d'élevage ne semble pas affectée par la sélection, contrairement à ce qui est suggéré dans la littérature de la théorie de l'allocation des ressources (Knap 2009). Finalement, les stratégies de sélection appliquées jusqu'à présent, dans un système d'alimentation ad libitum et sur la base de rations alimentaires faciles à digérer, n'ont pas affecté l'efficacité digestive des porcs pendant la croissance. Une amélioration 
génétique de l'efficacité digestive pourrait cependant être un élément de compétitivité pour la valorisation de ressources alimentaires riches en fibres, moins onéreuses que les matières premières utilisées classiquement en alimentation animale. Un certain nombre de défis s'ouvre donc pour poursuivre l'amélioration de ce caractère tout en répondant à l'évolution des contraintes de l'élevage, en particulier les exigences de multiperformance et de durabilité.

Dans ce contexte, deux leviers sont d'importance pour la filière porcine : le levier économique (l'aliment y contribue à $70 \%$ ), qui devra être réexaminé à la lumière de la disponibilité de nouvelles ressources alimentaires moins facilement valorisables par les animaux (voir plus loin), et le levier environnemental, qui reste un enjeu majeur pour la filière. Il n'y a pas actuellement de méthodologies à coût raisonnable permettant une quantification directe individuelle de l'excrétion des animaux en conditions d'élevage, qui permettrait une sélection de ce caractère. Les estimations effectuées s'appuient sur des équations de prédiction moyennes à partir de l'ingéré et des performances des animaux, tel que décrit dans la partie 1.2.d. Elles sont fortement corrélées avec l'indice de consommation, et ne tiennent pas compte de la variabilité individuelle des animaux à retenir chaque type de nutriment. Parmi les nouveaux outils qui permettraient de surmonter ces limites et de dégager des stratégies de sélection, on peut citer les techniques en cours de développement permettant de mesurer la digestibilité des nutriments dans les fèces à partir de prélèvements ponctuels en utilisant la spectrométrie infrarouge. De façon complémentaire, les méthodologies d'analyse de cycle de vie permettront d'évaluer l'impact environnemental des combinaisons de génétique et de conduite des animaux, en quantifiant explicitement différents critères, tels que l'eutrophisation de l'eau et l'acidification des sols.

On peut dans cette perspective revenir à la définition de l'efficacité alimentaire, et aux critères utilisés pour la mesurer. Les travaux évoqués plus haut concernent la consommation moyenne journalière résiduelle, qui cible explicitement la partie de l'ingéré qui ne dépend pas des performances de production et des besoins d'entretien moyens. Cette mesure, par rapport à l'IC, présente l'avantage majeur pour l'amélioration génétique d'être une combinaison linéaire de caractères, alors que l'IC est un rapport. En effet, la théorie de la sélection suppose la linéarité entre les composantes d'un index, et les évaluations de réponse à la sélection pour des rapports sont par conséquent erronées, ou approximées. Dans ce sens, la CMJR présente un intérêt majeur pour le sélectionneur. Cependant, il convient de réaliser que l'IC reste la mesure économique directe de l'efficacité alimentaire à l'échelle de l'animal. De plus, le calcul de la CMJR tel que proposé par Koch et al (1963) suppose un coût alimentaire identique pour les différents facteurs de l'équation quels que soient les animaux. Par exemple, l'équation utilisée dans l'expérience de sélection de l'INRA suppose un coût alimentaire de 1,06 g de CMJ par gramme de GMQ de l'animal (voir équation de l'encadré 2). Il est réaliste de penser que, à ration alimentaire donnée, ce coût dépend partiellement de la génétique de l'animal. Des approches statistiques de type régression aléatoire pourraient capter cette variabilité individuelle, ce qui permettrait de sélectionner spécifiquement pour le coût de chaque fonction.

Ces deux mesures, IC et CMJR, devront à termes, si la nature des rations alimentaires se diversifie, être exprimées en unité d'énergie pour une meilleure comparaison de résultats entre études, comme c'est souvent le cas chez les ruminants. L'énergie est en effet le levier principal de la réalisation des performances des porcs en élevage, même si ceux-ci sont capables au moins partiellement d'ajuster leur consommation alimentaire à la densité énergétique de la ration. De nouvelles études envisagent cependant de s'intéresser en complément à l'efficacité d'utilisation d'autres nutriments, tels que l'azote et le phosphore, voire les minéraux et les vitamines. Dans cet esprit, une première étude menée en 2012 avait suggéré une faible variabilité d'origine génétique de l'efficacité du dépôt protéique chez le porc en croissance en condition d'élevage standard (Brossard et al 2012b). Pour affiner ces calculs d'efficacité alimentaire globale pendant la croissance, certaines études s'intéressent à l'exploitation des données hautdébit disponibles grâce aux automates d'alimentation individuels soit pour décrire plus précisément la dynamique de l'efficacité alimentaire du porc au cours de

Figure 9. Impact de la sélection pour une CMJR réduite chez le porc en croissance sur les performances et les fonctions métaboliques majeures identifié à partir des expérimentations conduites sur des lignées divergentes de porcs Large White.

\section{EFFICACITÉ DIGESTIVE}

Pas de changement

Modifications du microbiote intestinal suggérées

\section{ACTIVITÉ/COMPORTEMENT ALIMENTAIRE}

Diminution du temps debout

Réduction des interactions avec les congénères

Réduction de l'activité alimentaire

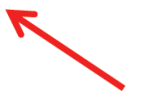

Sélection pour CMJR réduite

Plus de muscle

Vitesse de croissance légèrement réduite
LACTATION

Pas de désavantage identifié

\section{MÉTABOLISME ÉNERGÉTIQUE}

Peu dinteraction sur la réponse à des ressources énergétiques variées impact sur le métabolisme énergétique du muscle modification du bilan énergétique
MÉTABOLISME BASAL

Diminution de la production de chaleur à jeun Modification de la distribution des lipides en fonction des sources alimentaires Besoins d'entretien réduits pour le tube digestif
Qualité technologique de la viande diminuée
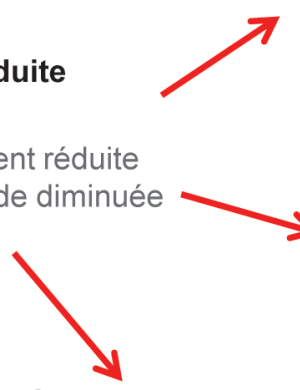

RÉPONSE À DES STRESS (chaleur, inflammation)

Pas de désavantage identifié

\section{MÉTABOLISME PROTÉIQUE}

Pas de conclusion claire

Augmentation des besoins en AA/kg d'aliment 
sa croissance (par exemple, voirHuynhTran et al 2017), soit pour explorer le comportement alimentaire des animaux les plus efficaces et identifier de nouveaux leviers de sélection.

La majorité des considérations précédentes s'appliquent aisément aux populations en sélection à objectifs paternels, telles que le Piétrain et les lignées mâles composites. En revanche, la sélection de la CMJ et l'efficacité alimentaire dans les lignées maternelles continue à faire débat dans la communauté scientifique. Si à l'INRA et sur les lignées américaines des profils de truies à meilleures performances en lactation ont été identifiés dans les lignées les plus efficaces, des craintes persistent quant à la capacité des truies qui présentent un ingéré réduit en lactation à faire face à des accroissements de taille et de poids de portée. Des études plus récentes confortent cependant l'hypothèse qu'une bonne capacité à mobiliser les réserves corporelles, puis à les reconstituer en début de gestation suivante, serait une clé de la capacité des truies à mener à bien leur lactation (Strathe et al 2017). Pour les lignées maternelles, des études plus poussées à l'échelle de carrières complètes intégrant gestations et lactations successives sont maintenant nécessaires pour trouver une mesure qui caractérise l'efficacité alimentaire de la truie dans une perspective de la longévité des carrières.

L'identification de marqueurs génomiques ou de biomarqueurs phénotypiques précoces de l'efficacité alimentaire utilisables en élevage reste une voie d'avenir pour sélectionner plus efficacement les porcs, quel que soit le critère retenu. L'utilisation de la génomique pour l'amélioration des populations porcines est une piste prometteuse, mais dont les coûts doivent être évalués en regard des coûts de phénotypage, et notamment de la mesure de l'ingéré alimentaire sur un nombre important d'animaux. Un avantage majeur de la génomique pourrait être l'évaluation des animaux sélectionnés non plus seulement pour leurs performances en race pure en élevage de sélection, mais pour les performances de leurs descendants commerciaux croisés élevés en élevages de production (Knol et al 2016).
La majorité des élevages porcins est actuellement conduite en lots homogènes en âge, éventuellement sexés, auxquels sont délivrés des quantités d'aliment légèrement restreintes à l'échelle du groupe. La pratique de la restriction a été à l'origine généralisée pour augmenter la teneur en maigre de la carcasse. Le contexte actuel est sensiblement différent : les porcs produits sont de plus en plus maigres, et l'arrêt de la castration des porcs mâles en 2018 dans l'Union Européenne va accentuer ce phénomène, les mâles entiers étant plus maigres que les castrés. La restriction appliquée à des types génétiques maigres entraîne une réduction simultanée des dépôts de protéines et de lipides, sans amélioration significative de l'IC. Elle n'est donc pas nécessairement justifiée dans ce nouveau contexte. L'ensemble de ces éléments suggère de faire évoluer les pratiques d'élevage pour ajuster au mieux les rations au potentiel des animaux grâce aux stratégies d'alimentation de précision.

Pour réduire la compétition avec la production de céréales pour l'alimentation animale et les biocarburants, une option consiste à introduire dans l'alimentation animale des ressources végétales de moindre qualité énergétique et nutritionnelle, ou non utilisables en alimentation humaine. Ces produits présentent généralement des proportions de fibres alimentaires plus importantes que les matières premières standards. La capacité spontanée des porcs à dégrader les fibres n'a cependant pas été améliorée, car à ce jour, la sélection n'expose pas les animaux à ces régimes alimentaires (Noblet et al 2013). L'évaluation en élevage de l'efficacité digestive devrait être bientôt possible grâce à la mise au point de techniques de phénotypage qui permettront d'évaluer sa variabilité génétique, ainsi que l'amplitude des interactions génétique $\mathrm{x}$ régime alimentaire dans les populations porcines. Dans ce contexte, un acteur jusque-là largement ignoré est le microbiote intestinal. C'est un partenaire à part entière de l'animal, contribuant à la fonction de barrière du tube digestif et à la transformation des aliments en composants absorbables par le tractus digestif. Des études sont nécessaires pour évaluer l'impact du microbiote intestinal sur la variabilité de l'effi- cacité alimentaire des porcs, et décomposer cet impact entre la fraction dépendante de la génétique de l'animal et la fraction dépendante des conditions d'élevage (conduite alimentaire, sanitaire...). Si nécessaire, des méthodes de phénotypage haut débit de la flore intestinale devront alors être proposées pour utiliser cette information en élevage, par exemple pour l'élaboration de probiotiques adaptés à des rations riches en fibres alimentaires, pour l'orientation des animaux vers des ressources alimentaires mieux adaptées au potentiel de leur flore (élevage de précision), ou pour la co-sélection d'animaux et de microbiote intestinal adaptés à des systèmes de production dédiés.

Finalement, un enjeu majeur de la filière porcine reste la capacité des animaux à maintenir des performances élevées en environnement de production changeant et parfois dégradé, c'est-àdire leur robustesse. L'environnement d'élevage est par essence variable, en lien avec les saisons, la qualité de l'aliment et de l'eau, la pression en pathogènes et l'état sanitaire du troupeau. L'environnement d'élevage offert aux animaux se diversifie en fonction des contraintes locales et de choix techniques des éleveurs. La quantification de la sensibilité des animaux aux variations de ces conditions permettrait de produire des indicateurs de robustesse. De premières études montrent que les porcs CMJRont un niveau d'ingéré et une vitesse de croissance moins affectés par la qualité de l'environnement que des porcs $\mathrm{CMJR}^{+}$(Gilbert et al 2014). Ces résultats rejoignent les études de réponses à des challenges évoquées dans ce document, ainsi que les résultats rapportés de façon indépendante dans l'expérience de sélection américaine mentionnée plus haut. Ils doivent être confirmés et mieux compris, mais suggèrent une interaction entre robustesse et efficacité alimentaire favorable pour la sélection, contrairement à la théorie de l'allocation des ressources appliquée aux animaux d'élevage. Si ces résultats n'étaient pas confirmés, des études complémentaires seraient nécessaires pour proposer des stratégies de sélection qui n'affectent pas la capacité des animaux à maintenir leur niveau de performance quand les conditions d'élevage sont dégradées. 


\section{Références}

Arkfeld E.K., Young J.M., Johnson R.C., Fedler C.A., Prusa K., Patience J.F., Dekkers J.C.M., Gabler N.K., Lonergan S.M., HuffLonergan E., 2015. Composition and quality characteristics of carcasses from pigs divergently selected for residual feed intake on high- or low-energy diets. J. Anim. Sci., 93, 2530-2545.

Barea R., Dubois S., Gilbert H., Sellier P., van Milgen J., Noblet J., 2010. Energy utilization in pigs selected for high and low residual feed intake. J. Anim. Sci., 88, 2062-2072.

Boddicker N., Gabler N.K., Spurlock M.E., Nettleton D., Dekkers J.C.M., 2011. Effects of ad libitum and restricted feed intake on growth performance and body composition of Yorkshire pigs selected for reduced residual feed intake. J. Anim. Sci., 89, 40-51.

Brossard L., Gilbert H., Billon Y., van Milgen J., 2012a. Effet d'une sélection divergente pour la consommation journalière résiduelle chez le porc en croissance sur la réponse à une carence en acides aminés. Journ. Rech. Porcine, 44, 165-170.

Brossard L., Gilbert H., van Milgen J., Noblet J., 2012b. Variabilité génétique de l'efficacité de dépôt protéique chez le porc en croissance : un potentiel limité pour la sélection? Journ. Rech. Porcine, 44, 47-48.

Bunger L., MacLeod M.G., Wallace C.A., Hill W.G., 1998. Direct and correlated effects of selection for food intake corrected for BW in the adult mouse. In: Proc. $6^{\text {th }}$ World Congr. Genet. Appl. Livest. Prod., 11-16 January 1998, Armidale, Australia, 97-100.

Bunter K.L., Hermesch S., Luxford B.G., Graser H.U., Crump R.E., 2005. Insulin-like growth factor-I measured in juvenile pigs is genetically correlated with economically important performance traits. Aust. J. Exp. Agr., 45, 783-792.

Bunter K.L., Cai W., Johnston D.J, Dekkers J.C.M., 2010. Selection to reduce residual feed intake in pigs produces a correlated response in juvenile insulin-like growth factor-I concentration. J. Anim. Sci., 88, 1973-1981.

Cai W., Casey D.S., Dekkers J.C.M., 2008. Selection response and genetic parameters for residual feed intake in Yorkshire swine. J. Anim. Sci., 86, 287-298.

Cai W., Kaiser M.S., Dekkers J.C.M., 2011. Genetic analysis of longitudinal measurements of performance traits in selection lines for residual feed intake in Yorkshire swine. J. Anim. Sci., 89, 1270-1280.

Cai W., Kaiser M.S., Dekkers J.C.M., 2012. Bayesian analysis of the effect of selection for residual feed intake on growth and feed intake curves in Yorkshire swine. J. Anim. Sci., 90, 127-141.

Campos P.H., Noblet J., Jaguelin-Peyraud Y., Gilbert H., Mormede P., de Oliveira Donzele R.F., Donzele J.L., Renaudeau D., 2014. Thermoregulatory responses during thermal acclimation in pigs divergently selected for residual feed intake. Int. J. Biometeorol., 58, 1545-1557.

Chatelet A., Merlot E., Gilbert H., Gondret F., Friggens N., Le Floc'h N., 2017. Impact of hygiene of housing conditions on performance and health of two pig genetic lines divergent for residual feed intake. Animal, doi:10.1017/ S1751731117001379.

Cruzen S.M., Harris A.J., Hollinger K., Punt R.M., Grubbs J.K., Selsby J.T., Dekkers J.C.M. Gabler N.K., Lonergan S.M., Huff-Lonergan E., 2013. Evidence of decreased muscle protein turnover in gilts selected for low residual feed intake. J. Anim. Sci., 91, 4007-4016.

Faure J., Lebret B., Brossard L., Billon Y., Lefaucheur L., Gilbert H., 2012. Vers une sélection sur la consommation alimentaire résiduelle chez le porc en croissance pour concilier efficacité alimentaire, qualité des viandes et impact environnemental. Journ. Rech. Porcine, $44,25-30$

Faure J., Lefaucheur L., Bonhomme N., Ecolan P. Meteau K. Coustard S.M. Kouba M. Gilbert H., Lebret B., 2013. Consequences of divergent selection for residual feed intake in pigs on muscle energy metabolism and meat quality. Meat Sci., 93, 37-45.

Fontanesi L., Schiavo G., Galimberti G., Calò D.G., Russo V., 2014. A genome wide association study for average daily gain in Italian Large White pigs. J. Anim. Sci., 92, 1385-1394.

Gabarrou J.F., Geraert P.A., Picard M., Bordas A., 1997. Diet-induced thermogenesis in cockerels is modulated by genetic selection for high or low residual feed intake. J. Nutr., 127, 2371-2376.

Gilbert H., Bidanel J.P., Gruand J., Caritez J.C., Billon Y., Guillouet P., Lagant H., Noblet J., Sellier P., 2007. Genetic parameters for residual feed intake in growing pigs, with emphasis on genetic relationships with carcass and meat quality traits. J. Anim. Sci., 85, 3182-3188.

Gilbert H., Alain S., Sellier P., Lagant H., Billon Y., Bidanel J.P., Guillouet P., Noblet J, van Milgen J., Brossard L., 2009a. Relations génétiques entre efficacités alimentaire et cinétiques de croissance et d'ingestion chez le porc Large-White. Journ. Rech. Porcine, 41, 1-6.

Gilbert H., Alain S., Bidanel J.P., Lagant H., Billon Y., Guillouet P., Noblet J., Sellier P., 2009 b. Sélection divergente sur la consommation alimentaire résiduelle du porc en croissance : effets corrélatifs sur le comportement alimentaire. Journ. Rech. Porcine, 41, 31-32.

Gilbert H., Bidanel J.P., Billon Y., Méteau K., Guillouet P., Noblet J., Sellier P., Gatellier P., Sayd T., Faure J., Lebret B., 2012a. Responses to divergent selection for residual feed intake in growing pigs, consequences on pork. $63^{\text {th }}$ EAAP Meeting, Bratislava, Slovakia, pp. 276.

Gilbert H., Billon Y., Fleury J., Noblet J., Gourdine J.L., Renaudeau D., 2012b. Are responses to selection in lines divergently selected for residual feed intake in growing pigs affected by GxE interactions when bred in a tropical environment? AnGR-NordicNET Workshop, Genotype-by-Environment Interactions and Adaptation of Farm Animals on Phenotypic and Molecular Levels, Tuusula, Finnland, 26-27.

Gilbert H., Bidanel J.P., Billon Y., Lagant H., Guillouet P., Sellier P., Noblet J., Hermesch S., 2012c. Correlated responses in sow appetite, residual feed intake, body composition, and reproduction after divergent selection for resi- dual feed intake in the growing pig. J. Anim. Sci., 90, 1097-1108.

Gilbert H., David I., Billon Y., Hermesch S., 2014. Does selection for RFI affect the sensitivity to environmental variation in pigs? 10th WCGALP. Vancouver, Canada. Paper 123.

Gilbert H., Billon Y., Brossard L., Faure J., Gatellier P., Gondret F., Labussière E., Lebret B., Lefaucheur L., Le Floc'h N., Louveau I., Merlot E., Meunier-Salaün M.C., Montagne L., Mormède P., Renaudeau D., Riquet J., RogelGaillard C., van Milgen J., Vincent A., Noblet J., 2017a. Review: divergent selection for residual feed intake in the growing pig. Animal, $11,1427-1439$

Gilbert H., Louveau I., Billon Y., Bunter K.L., 2017b. Juvenile IGF-I response in INRA RFI selection lines partly reflects changes in postweaning attributes. In : Assoc. Adv. Anim. Breed. Genet., Rydges Southbank Townsville, QLD, AUS (2017-07-02 - 2017-07-05) https://prodinra.inra.fr/record/394694

Gondret F., Louveau I., Mourot J., Duclos M.J., Lagarrigue S., Gilbert H., van Milgen J., 2014. Dietary energy sources affect the partition of body lipids and the hierarchy of energy metabolic pathways in growing pigs differing in feed efficiency. J. Anim. Sci., 92, 4865-4877.

Gondret F., Vincent A., Houée-Bigot M., Siegel A., Lagarrigue S., Causeur D., Gilbert H., Louveau I., 2017. A transcriptome multi-tissue analysis identifies biological pathways and genes associated with variations in feed efficiency of growing pigs. BMC Genom., 18, 244.

Grubbs J.K., Fritchen A.N., Huff-Lonergan E., Dekkers J.C.M., Gabler N.K., Lonergan S.M., 2013. Divergent genetic selection for residual feed intake impacts mitochondria reactive oxygen species production in pigs. J. Anim. Sci., 91, 2133-2140.

Grubbs J.K., Lonergan S.M., Dekkers J.C.M., Tuggle C.K., 2014. Identification of potential serum biomarkers for feed efficiency in young pigs. J. Anim. Sci., 92(E-Suppl 2), 187.

Grubbs J.K., Dekkers J.C.M., Huff-Lonergan E. Tuggle C.K., Lonergan S.M., 2016. Identification of potential serum biomarkers to predict feed efficiency in young pigs. J. Anim. Sci., 94, 1482-1492.

Harris A.J., Patience J.F., Lonergan S.M., Dekkers J.C.M., Gabler N.K., 2012. Improved nutrient digestibility and retention partially explains feed efficiency gains in pigs selected for low residual feed intake. J. Anim. Sci., 90, 164-166.

Hauptli L., Priet A., Gilbert H., Montagne L., 2013. Response to a high level fibers diet in pigs divergently selected for residual feed intake. In: Book Abstr. 64th Ann. Meet. Eur. Assoc. Anim. Prod., 26-30 August 2013, Nantes, France, pp 589.

Herd R.M., Arthur P.F., 2009. Physiological basis for residual feed intake. J. Anim. Sci., 87, E64-E71.

Hermesch S., Li L., Doeschl-Wilson A., Gilbert H., 2015. Selection for productivity and robustness traits in pigs. Anim. Prod. Sci., 55, 1437-1447. 
Hoque M.A., Katoh K., Suzuki K., 2009. Genetic associations of residual feed intake with serum insulin-like growth factor-I and leptin concentrations, meat quality, and carcass cross sectional fat area ratios in Duroc pigs. J. Anim. Sci., 87, 3069-3075.

Huynh-Tran V.H., Gilbert H, David I., 2017. Genetic structured antedependence and random regression models applied to the longitudinal feed conversion ratio in growing Large White pigs. J. Anim. Sci., 95, 4752-4763.

IFIP, 2016-2017. Le porc par les chiffres. Ed. IFIP.

IFIP-GTE, 2014. GTE : évolution des résultats moyens nationaux - naisseurs - engraisseurs Ed IFIP.

Jégou M., Gondret F., Martin-Lalande J., Tea I. Baéza E., Louveau I., 2016a. NMR-based metabolomics highlights differences in plasma metabolites in pigs exhibiting diet-induced differences in adiposity. Eur. J. Nutr., 55, 1189 1199.

Jégou M., Gondret F., Vincent A., Tréfeu C., Gilbert H., Louveau I., 2016b. Whole blood transcriptomics is relevant to identify molecular changes in response to genetic selection for feed efficiency and nutritional status in the pig. PLoS One, 11: e0146550 (DOI 10.1371/journal.pone.0146550).

Knap P.W., 2009. Allocation of resources to maintenance. In Resource allocation theory applied to farm animal production. Rauw W.M. (Eds). 210-229. CABI Publishing, Wallingford,

Knol E.F., Nielsen B., Knap P.W., 2016 Genomic selection in commercial pig breeding. Anim. Front., 6, 15-22.

Koch R.M., Swiger L.A., Chambers D., Gregory K.E., 1963. Efficiency of feed use in beef cattle. J. Anim. Sci., 22, 486-494.

Labussière E.., Dubois S., Gilbert H., Thibault J.N., Le Floc'h N., Noblet J., van Milgen J., 2015. Effect if inflammation stimulation on energy and nutrient utilization in piglets selected for low and high residual feed intake. Animal, 9, 1653-1661.

Lebret B., 2004. Rationalization of pig production: consequences on meat quality. INRA Prod. Anim., 17, 79-91.

Lefaucheur L., Lebret B., Ecolan P., Louveau I., Damon M., Prunier A., Billon Y., Sellier P. Gilbert H., 2011. Muscle characteristics and meat quality traits are affected by divergent selection on residual feed intake in pigs. J. Anim. Sci., 89, 996-1010.

Le Naou T., Le Floc'h N., Louveau I., Gilbert H., Gondret F., 2012. Metabolic changes and tissue responses to selection on residual feed intake in growing pigs. J. Anim. Sci., 90, 47714780

Le Roith D., Bondy C. Yakar S., Liu J.L., Butler A., 2001. The somatomedin hypothesis. Endocr. Rev., 22, 53-74.

Liu H., Nguyen Y., Nettleton D., Dekkers J.C.M., Tuggle C.K., 2015. Identification of early blood differentially expressed genes between two pig lines divergently selected for feed efficiency: potential biomarkers for feed efficiency. $48^{\text {th }}$ ASAS Midwestern Sect. Meeting, des Moines, USA, pp. 106.
Louveau I., Vincent A., Tacher S., Gilbert H., Gondret F., 2016. Increased expressions of genes and proteins involved in mitochondrial oxidation and antioxidant pathway in adipose tissue of pigs selected for a low residual feed intake. J. Anim. Sci., 94, 5042-5054.

Luiting P., Urff E.M., Verstegen M.W.A., 1994 Between-animal variation in biological efficiency as related to residual feed consumption. Neth. J. Agric. Sci., 42, 59-67.

Mauch E.D., Gabler N.K., Weber T.E., Patience J.F., Kerr B.J., Dekkers J.C.M., 2015. The effect of divergent selection for residual feed intake on digestibility of control and low energy, high fiber diets. $48^{\text {th }}$ ASAS Midwestern Sect. Meeting, des Moines, USA, pp. 46.

Merlot E., Evrard J., Vincent A., Gilbert H., Le Floc'h N., 2011. Effects of a divergent selection for residual feed intake on nutrient metabolism and immune response during an inflammatory challenge. Oskar Kellner Symposium, Warnemunde, Germany, pp. 89

Merlot E., Gilbert H., Le Floc'h N., 2016 Metabolic response to an inflammatory challenge in pigs divergently selected for residual feed intake. J. Anim. Sci., 94, 563-573.

Meunier-Salaün M.C., Guérin C., Billon Y. Sellier P., Noblet J., Gilbert H., 2014. Divergen selection for residual feed intake in grouphoused growing pigs: characteristics of physical and behavioural activity according to line and sex. Animal, 8, 1898-1906.

Montagne L., Loisel F., Le Naou T., Gondre F., Gilbert H., Le Gall M., 2014. Difference in short-term responses to a high-fiber diet in pigs divergently selected for residual feed intake. J Anim. Sci., 92, 1512-1523.

Noblet J., Karege C., Dubois S., van Milgen J., 1999. Metabolic utilisation of energy and maintenance requirements in growing pigs: effect of sex and genotype. J. Anim. Sci., 77, 1208-1216.

Noblet J., Gilbert H., Jaguelin-Peyraud Y. Lebrun T., 2013. Evidence of genetic variability for digestive efficiency in the growing pig fed a fibrous diet. Animal, 7, 1259-1264.

Onteru S.K., Gorbach D.M., Young J.M., Garrick D.J., Dekkers J.C.M., Rothschild M.F. 2013. Whole genome association studies of residual feed intake and related traits in the pig. PLoS ONE, 8, e61756. doi:10.1371/journal.pone. 0061756

Pastorelli H., Prunier A., Brossard L., Montagne L., Gilbert H., Larzul C., Merlot E., 2015. Evaluation of relations between health, death and growth in growing pigs. Journ. Rech. Porcine, 47, 57-62.

Prunier A., Heinonen M., Quesnel H., 2010. High physiological demands in intensively raised pigs: impact on health and welfare. Animal, 4, 886-898.

Renaudeau D., Frances G., Dubois S., Gilbert H., Noblet J., 2013. Effect of thermal heat stress on energy utilization in two lines of pigs divergently selected for residual feed intake. J. Anim. Sci., 91, 1162-1175.

Renaudeau D., Gourdine J.L., Fleury J., Ferchaud S, Billon Y, Noblet J, Gilbert H. 2014. Selection for residual feed intake in growing pigs: Effects on sow performance in a tropical climate. J. Anim. Sci., 92, 3568-3579.
Riquet J., Labrune Y., Feve K., Billon Y., Gilbert H., 2014. Whole genome characterization and associations studies in two divergent pig lines selected on residual feed intake. $10^{\text {t }}$ WCGALP, Vancouver, Canada, poster \#571.

Rolf M.M., Taylor J.F., Schnabel R.D., McKay S.D., McClure M.C., Northcutt S.L., Kerley M.S., Weaber R.L., 2012. Genome-wide association analysis for feed efficiency in Angus cattle. Animal, Genet., 43, 367-74.

Sadler L.J., Johnson A.K., Lonergan S.M., Nettleton D., Dekkers J.C.M., 2011. The effect of selection for residual feed intake on general behavioural activity and the occurrence of lesions in Yorkshire gilts. J. Anim. Sci., 89 258-266.

Saintilan R., Mérour I., Brossard L., Tribout T., Dourmad J.Y., Sellier P., Bidanel J., van Milgen J., Gilbert H., 2013. Genetics of residual feed intake in growing pigs: relationships with production traits, and nitrogen and phosphorus excretion. J. Anim. Sci., 91, 2542-2554.

Saintilan R., Brossard L., Vautier B., Sellier P. Bidanel J., van Milgen J., Gilbert H., 2015. Phenotypic and genetic relationships between growth and feed intake curves and feed efficiency and amino acid requirements in the growing pig. Animal, 9, 18-27.

Santana M.H., Utsunomiya Y.T., Neves H.H., Gomes R.C., Garcia J.F., Fukumasu H., Silva S.L., Oliveira Junior G.A., Alexandre P.A., Leme P.R., Brassaloti R.A., Coutinho L.L., Lopes T.G., Meirelles F.V., Eler J.P., Ferraz J.B., 2014. Genome-wide association analysis of feed intake and residual feed intake in Nellore cattle. BMC Genet., 11, 15-21.

Shirali M., Doeschl-Wilson A., Knap P.W., Duthie C., Kanis E., van Arendonk J.A.M., Roehe R., 2012. Nitrogen excretion at different stages of growth and its association with production traits in growing pigs. J. Anim. Sci., 90, 1756-1765

Shirali M., Doeschl-Wilson A., Duthie C. Knap P.W., Kanis E., van Arendonk J.A.M., Roehe R., 2014. Estimation of residual energy intake and its genetic background during the growing period in pigs. Livest. Sci., 168, 17-25.

Smith R.M., Gabler N.K., Young J.M., Cai W., Boddicker N.J., Anderson M.J., Huff-Lonergan E., Dekkers J.C.M., Lonergan S.M., 2011. Effects of selection for decreased residual feed intake on composition and quality of fresh pork. J. Anim. Sci., 89, 192-200.

Strathe A.V., Bruun T.S., Hansen C.F., 2017. Sows with high milk production had both a high feed intake and high body mobilization. Animal, 11, 1913-1921.

Swennen Q., Verhulst P.J., Collin A., Bordas A., Verbeke K., Vansant G., Decuypere E., Buyse J., 2007. Further investigations on the role of diet-induced thermogenesis in the regulation of feed intake in chickens: comparison of adult cockerels of lines selected for high or low residual feed intake. Poultr. Sci., 86, 1960-1971.

Tribout T., Caritez J.C., Gogue J., Gruand J., Bouffaud M., Billon Y., Pery C., Griffon H., Brenot S., Le Tiran M.H., Bussieres F., Le Roy P., Bidanel J.P., 2004. Estimation, par utilisation de semence congelée, du progrès génétique réalisé en France entre 1977 et 1998 dans la race porcine Large White : résultats pour quelques caractères de production et de qualité 
des tissus gras et maigres. Journ. Rech. Porcine, $36,275-282$

Vincent A., Louveau I., Gondret F., Tréfeu C., Gilbert H., Lefaucheur L., 2015. Divergent selection for residual feed intake affects the transcriptomic and proteomic profiles of pig skeletal muscle. J. Anim. Sci., 93, 2745-2758
Young J.M., Cai W., Dekkers J.C.M., 2011. Effect of residual feed intake on feeding and daily feed intake patterns in Yorkshire swine. J. Anim. Sci., 89, 639-647.

Young J.M., Bergsma R., Knol E.F., Patience J.F., Dekkers J.C.M., 2016. Effect of selection for residual feed intake during the grow/finish phase of production on sow reproduction performance and lactation efficiency. J. Anim. Sci. 94, 1420-4132.

\section{Résumé}

L'amélioration de l'efficacité alimentaire est un enjeu majeur pour la compétitivité de la filière porcine. Elle est classiquement mesurée en élevage par l'indice de consommation, indicateur économique de l'efficacité d'utilisation des aliments pour la croissance. Un critère alternatif indépendant des performances des animaux, la Consommation Moyenne Journalière Résiduelle (CMJR), a été proposé dans les années 1960. Cet article synthétise les résultats obtenus à partir de la sélection divergente de deux lignées à l'INRA, une lignée à CMJR faible, animaux consommant moins que prédit pour leurs besoins de production et d'entretien, et donc plus efficaces, et une lignée à CMJR élevée, animaux consommant plus que prédit pour leurs besoins, moins efficaces. Ces recherches montrent qu'il est possible de sélectionner pour la CMJR chez le porc en croissance avec un impact limité sur les caractères de production et de reproduction femelle, permettant ainsi de diminuer les quantités d'aliments utilisées en élevage. La dégradation des paramètres de qualité technologique de la viande en réponse à l'amélioration génétique de l'efficacité alimentaire est un point de vigilance dans les stratégies de sélection, de même que la composition nutritionnelle de la ration alimentaire. Finalement, la capacité de réponse à des stress ou à des changements de conditions d'élevage ne semble pas affectée par la sélection, contrairement à ce qui est suggéré dans la littérature en réponse à la sélection pour une CMJR réduite. Les défis qui résultent de ces résultats pour poursuivre l'amélioration de ce caractère en production tout en répondant à l'évolution des contraintes de l'élevage sont discutés.

\section{Abstract \\ Selection for residual feed intake in pigs: impacts on production traits and challenges for pork production}

Improving feed efficiency remains a challenge for pork production. The usual measure in herds is feed conversion ratio that quantifies the economic efficiency of feed use for animal growth. Residual Feed Intake (RFI) has been proposed as an alternative measure, independent of production traits, in the 1960's. This paper reviews the research in two divergent lines selected for RFI at INRA, the low RFI line with pigs eating less than predicted from the production and maintenance requirements, i.e. more efficient, and the high RFI line eating more than predicted for its less efficient requirements,. These studies show that selection for RFI in growing pigs is possible with a limited impact on production and sow reproduction. However, technological meat quality is unfavourably affected by selection and a control of the impacts of selection for feed efficiency on this trait is necessary, as well as for nutritional requirements of most efficient pigs. Finally, the pig's ability to respond to stressors or various breeding conditions was not affected by selection, unlike that hypothesized by the resources allocation theory applied to livestock species. The challenges for pig production to continue improving feed efficiency in a changing context for livestock production are discussed.

GILBERT H., BILLON Y., BROSSARD L., FAURE J., GATELLIER P., GONDRET F., LABUSSIÈRE E., LEBRET B., LEFAUCHEUR L., LE FLOCH N., LOUVEAU I., MERLOT E., MEUNIER-SALAÜN M.-C., MONTAGNE L., MORMĖDE P., RENAUDEAU D., RIQUET J., ROGEL-GAILLARD C., VAN MILGE J., VINCENT A., NOBLET J., 2017. Sélection pour la consommation alimentaire moyenne journalière résiduelle chez le porc : impacts sur les caractères et défis pour la filière. INRA Prod. Anim., $30,439-454$. 
